\title{
Spatio-temporal organization in a morphochemical electrodeposition model: Hopf and Turing instabilities and their interplay
}

\author{
DEBORAH LACITIGNOLA ${ }^{1}$, BENEDETTO BOZZINI $^{2}$ and \\ IVONNE SGURA ${ }^{3}$ \\ ${ }^{1}$ Dipartimento di Ingegneria Elettrica e dell'Informazione, Università di Cassino e del Lazio Meridionale, \\ Via di Biasio, I-03043 Cassino, Italy \\ email: d.lacitignola@unicas.it \\ ${ }^{2}$ Dipartimento di Ingegneria dell'Innovazione, Università del Salento, via Monteroni, I-73100 Lecce, Italy \\ email: benedetto.bozzini@unisalento.it \\ ${ }^{3}$ Dipartimento di Matematica e Fisica "E. De Giorgi”, Università del Salento, via per Arnesano, \\ I-73100 Lecce, Italy \\ email: ivonne.sgura@unisalento.it
}

(Received 20 November 2013; revised 12 November 2014; accepted 18 November 2014; first published online 22 December 2014)

\begin{abstract}
In this paper, we investigate from a theoretical point of view the $2 \mathrm{D}$ reaction-diffusion system for electrodeposition coupling morphology and surface chemistry, presented and experimentally validated in Bozzini et al. (2013 J. Solid State Electr. 17, 467-479). We analyse the mechanisms responsible for spatio-temporal organization. As a first step, spatially uniform dynamics is discussed and the occurrence of a supercritical Hopf bifurcation for the local kinetics is proved. In the spatial case, initiation of morphological patterns induced by diffusion is shown to occur in a suitable region of the parameter space. The intriguing interplay between Hopf and Turing instability is also considered, by investigating the spatio-temporal behaviour of the system in the neighbourhood of the codimensiontwo Turing-Hopf bifurcation point. An ADI (Alternating Direction Implicit) scheme based on high-order finite differences in space is applied to obtain numerical approximations of Turing patterns at the steady state and for the simulation of the oscillating Turing-Hopf dynamics.
\end{abstract}

Key words: Reaction-diffusion models; Pattern formation; Turing-Hopf instability; ADI method; Finite differences

\section{Introduction}

Alloy electrodeposition processes have been shown to exhibit electrokinetic instabilities that can lead to compositional heterogeneity in the bulk of electrodeposits. Starting from this evidence, a number of papers have insightfully investigated the formation of spatiotemporal structures from an experimental point of view, but the attempts to single out the factors leading to the development of specific patterns have hardly exceeded the empirical level. 
The notable circumstance that experimental patterns found in electrodeposition processes exhibit structures that are strongly reminiscent of those found in many different natural systems (such as fish and animal coat markings, sand dunes, grass landscapes) has suggested that such experimental electrochemical scenarios could be explained within the reaction-diffusion modelling framework [40,45].

In fact, since the pioneering intuition of Alan Turing [66], pattern formation in reactiondiffusion systems has become the paradigm for models of spatial self-organization and the question "How do these patterns arise?" has produced stimulating research in the field of non-linear dynamics [46,47,51]. As a matter of fact, Turing's diffusion-driven instability is now universally recognized as one of the leading mechanisms of spatial pattern formation in reaction-diffusion systems and the related Turing patterns have been the subject of extensive studies in a variety of applied contexts [20,21, 25, 28, 32, 46, 48, 51, 65].

In this regard, chemistry seems to have had a privileged role. As insightfully expressed in [68], the advantage of chemical systems over biological ones is that one can perform experiments under controllable conditions and manipulate the relevant parameters in order to generate patterns. Chemical systems can hence offer rigorous experimental validation of the theoretical predictions and can thereby probe fundamental issues about pattern formation. Turing patterns in a chemical reactor were first experimentally observed by De Kepper's group in a chlorite-iodide-malonic acid (CIMA) reaction [18] and later confirmed by Ouyang and Swinney who observed striped as well as spotty patterns [53]. The chance to experimentally detect Turing structures has produced a renewed interest in these systems as shown by the large number of theoretical $[35,58]$, computational $[43,44,67]$ and experimental studies in the field $[6,42,52]$.

The mathematical modelling of electrochemical dynamics and of the related pattern formation processes has been mainly developed for variants of the activator-inhibitor mechanism and focused on electrocatalysis [36,40,45] more than electrodeposition. Nevertheless, in this field experimental pattern formation has been observed in a series of experiments regarding, among others, Ag [27,38], Co-In [39] and Ni-P-W-Bi [15] alloys. Also the mathematical description of these phenomena has generally been developed within the reaction-diffusion modelling approach, with the rather artificial ansatz of considering exclusively concentrations of the reactive species as state variables for the electrodeposition models [26,37,59-61].

In a series of recent papers [7-15] and [63] we used the reaction-diffusion modelling approach to rationalize the formation of morphological patterns in electrodeposition. The novelty of our approach was to consider more natural state variables: the morphology (surface profile) - that is the crucial observable - and the surface chemistry (composition) - that in fact controls the growth process - coupled through non-linear and physically straightforward electrochemical source terms. The resulting two variable non-linear reaction-diffusion system led to the formation of Turing patterns as well as to the development of transition front waves and allowed us to gain a detailed understanding of the $3 \mathrm{D}$ electrocrystallisation process.

In this paper, we consider the spatially $2 \mathrm{D}$ reaction-diffusion system introduced in [15] and based on classical textbook electrokinetics, which notably improves our previous model studied in [9] that contained rather crude physico-chemical approximations. As 
shown in [15] by extensive numerical simulations, this new model is interestingly flexible in terms of accounting for typical electrokinetic control phenomena and includes all spatiotemporal organization types that, to the best of our efforts, we have been able to find in the experimental literature (see [15] and references therein for a comprehensive gallery of the experimental results). In [15] the spatial pattern initiation was not investigated from an analytical point of view and we wish to address this point in detail in the present paper: we show that our model supports spatial pattern initiation, because of Turing's diffusiondriven instability, and that it can also exhibit a variety of spatio-temporal phenomena. This feature of our model can be in part explained by observing that it displays interesting dynamics in the spatially uniform case since the physically relevant steady state can lose its stability via a transcritical bifurcation as well as via a supercritical Hopf bifurcation. This latter circumstance seems to be noteworthy because recent studies [2,3] have shown that the emergence of spatio-temporal patterns in reaction-diffusion systems can be related to the presence of unstable homogeneous states, like those found beyond a supercritical Hopf bifurcation.

The simultaneous appearance of Turing instability, which leads to steady spatial structures, with Hopf instability, which gives rise to temporal oscillations, is of great interest because these bifurcations are responsible for the breaking of spatial and temporal symmetries, respectively. The coupling between these two kinds of instabilities was first theoretically investigated in the 90's in the framework of the Lengyel-Epstein model of the CIMA reaction [56]. Since then it has become a key topic for pattern formation in the reaction-diffusion framework and has been extensively investigated in chemistry, physics and biology $[2,3,24,33,48,49,55]$.

The Turing-Hopf (TH) interaction can take place either due to different competing bifurcations of multiple stationary states $[23,25]$ or through a codimension-two TH bifurcation [24,57]. For small two-dimensional systems, the following scenarios are expected in the neighbourhood of a codimension-two TH bifurcation point: (i) a $\mathrm{TH}$ bistability regime, where either a stationary spatial pattern or a spatially uniform oscillating solution can be attained, depending on the initial conditions; (ii) a TH 2D mixed mode, such as stripes and hexagons, oscillating homogeneously in time with one frequency; (iii) a subharmonic Turing mode, resulting in a spatial pattern with two wavenumbers and one frequency; (iv) a subharmonic TH mode, which leads to spatial patterns with two wavenumbers and two frequencies. In addition, when large systems are considered, spatial modulations of the amplitude can lead to long-wavelength instabilities [25].

Both Turing and Hopf instabilities have been experimentally observed separately in the CIMA reaction by varying the concentration of the colour indicator in the reactor $[25,54]$. Moreover, in $[69,70]$ a variety of both stationary and oscillating structures were obtained in the numerical simulations of a system with interacting modes. On the other hand, due to the interaction between Turing and Hopf instabilities, oscillatory Turing patterns were also found in the Belousov-Zhabotinsky reaction in a water-in-oil microemulsion [34] and in the chlorine dioxide-iodine-malonic acid (CDIMA) reaction [50].

In the neighbourhood of a codimension-two $\mathrm{TH}$ point, chaotic $2 \mathrm{D}$ dynamics has also been observed both experimentally [5] and numerically [49] although a theoretical 
characterization of the mechanisms leading to chaos has only rarely been provided [1]. In this respect, within the ecological context, a fascinating debate is currently going on: see e.g. [2,3]. In [3] the authors use the concept of generalized models developed in [31] to show that in a modified diffusive predator-prey Rosenzweig-McArthur model, spatially irregular self-sustained non-stationary patterns or even spatio-temporal chaos can appear for parameter values in the neighbourhood of the $\mathrm{TH}$ bifurcation point. Instead, in [2], where a two species reaction-diffusion predator-prey system with a ratio-dependent functional response is considered, spatio-temporal chaos is not found in the close vicinity of the $\mathrm{TH}$ bifurcation, but rather for parameter values far from the bifurcation point. These two examples show how the occurrence of chaotic spatio-temporal dynamics near a TH point is still a controversial issue.

In the present paper, we will show that the spatio-temporal phenomenology occurring in the neighbourhood of a codimension-two TH point can be further enriched by focusing on the possible destabilization à la Turing of the limit cycle born by a supercritical Hopf bifurcation. In [55] this mechanism is referred to as TH instability or diffusive instability of the Hopf limit cycle and represents a different way in which Turing and Hopf instabilities can interact. We will exploit the analytical approach developed in [55], which employs perturbation expansions, to find appropriate normal modes useful for the study of the TH instability of the Hopf limit cycle. We show that, in the neighbourhood of the TH point, our system can exhibit both a classical $\mathrm{TH}$ bistability regime and a $\mathrm{TH}$ instability of the Hopf cycle which possibly results in oscillatory inhomogeneous patterns. The possible occurrence of $2 \mathrm{D}$ chaotic spatio-temporal dynamics is also discussed in this context. These theoretical findings will be illustrated through an extensive gallery of numerical simulations both for stationary and oscillating Turing patterns.

The structure of the paper is detailed below. In Section 2, we recall the main features of the 2D reaction-diffusion system introduced in [15]; a brief overview of the system properties in the spatially uniform case is provided in Section 3; the conditions for the emergence of spatial patterns via Turing instability are discussed in Section 4; higher codimension bifurcation points are treated in Section 5 and in Section 6 we investigate, from the analytical point of view, the destabilization à la Turing of the Hopf limit cycle and the resulting spatio-temporal dynamics in the neighbourhood of the codimension-two $\mathrm{TH}$ bifurcation point. In Section 7, we show the numerical simulations, some information about the numerical methods used and a qualitative discussion about the role of some parameters of crucial electrochemical interest in the selection of the typology of patterns obtained. Concluding remarks and possible ramifications of the obtained results are reported in Section 8.

\section{The model}

In this paper, we deepen the analysis of the reaction-diffusion model proposed in [15], whose key feature is the coupling of one equation for the morphology $\eta(x, y, t)$ with one for the surface chemistry $\theta(x, y, t) . \eta \in \mathbb{R}$ is adimensional and expresses the instantaneous increment of the electrodeposit profile. $0 \leqslant \theta(x, y, t) \leqslant 1$ is the surface coverage with the 
functionally crucial adsorbate. The PDE system in adimensional form is given by

$$
\begin{aligned}
& \frac{\partial \eta}{\partial t}=\Delta \eta+f(\eta, \theta), \\
& \frac{\partial \theta}{\partial t}=d \Delta \theta+g(\eta, \theta),
\end{aligned}
$$

where $\Delta$ is the two-dimensional Laplacian operator and $d=D_{\theta} / D_{\eta}$ is the ratio of the diffusion coefficients for the individual chemical and morphological processes, respectively. The non-linear source terms that account for generation (deposition) and loss (corrosion) of the relevant material are given by

$$
\begin{aligned}
& f(\eta, \theta)=A_{1}(1-\theta) \eta-A_{2} \eta^{3}-B(\theta-\alpha), \\
& g(\eta, \theta)=C\left(1+k_{2} \eta\right)(1-\theta)[1-\gamma(1-\theta)]-D\left[\theta(1+\gamma \theta)+k_{3} \eta \theta(1+\gamma \theta)\right] .
\end{aligned}
$$

Model (2.1)-(2.2) is defined for $(x, y, t) \in\left[0, L_{x}\right] \times\left[0, L_{y}\right] \times[0, T]$, with $L_{x}, L_{y}$ characteristic lengths of the electrode and $T$ a characteristic time of the electrodeposition process. We also require (2.1)-(2.2) to be supplemented by zero-flux boundary conditions and the following initial conditions:

$$
\eta(x, y, 0)=\eta_{0}(x, y), \quad \theta(x, y, 0)=\theta_{0}(x, y), \quad(x, y) \in\left[0, L_{x}\right] \times\left[0, L_{y}\right] .
$$

The physical meaning of the source terms (2.2) is briefly described here; full details are provided in [15]. The term $A_{1}(1-\theta) \eta$ accounts for the charge-transfer rate at sites free from adsorbates; $A_{2} \eta^{3}$ describes mass-transport limitations to the electrodeposition process. The term $-B(\theta-\alpha)$ quantifies the effect of adsorbates on the electrodeposition rate. The parameter $0<\alpha \leqslant 1$ takes into account the fact that adsorbates can have both inhibiting and enhancing effects on the growth rate. The source term $g$, that can be regarded as $g(\eta, \theta)=C g_{a d s}(\eta, \theta)-D g_{d e s}(\eta, \theta)$, features adsorption (parameter $C$ ) and desorption (parameter $D$ ) terms including both chemical (expanded to second order) and electrochemical (first order) contributions. Moreover, the source terms (2.2) contain two major improvements with respect to the ones used in [9]: (i) they are physically more straightforward, as their analytical form is fully compatible with classical theories of electrodeposition kinetics and (ii) they are more flexible because non-linearities and couplings can be switched on and off simply by properly choosing parameter values, thus allowing us to build a hierarchy of models from the same analytical concept.

For simplicity and without loss of generality, the results reported in this work are derived under the following assumptions: (i) all the constants are taken as real positive or equal to zero, with $0<\gamma \leqslant 1$; (ii) $k_{3}<k_{2}$, meaning that adsorption is the dominating chemical contribution to growth; (iii) the following relation holds:

$$
D=\frac{C(1-\alpha)(1-\gamma+\gamma \alpha)}{\alpha(1+\gamma \alpha)},
$$

implying that adsorbates enhance the growth rate and the adsorption and desorption rates are proportional $(D \propto C)$. The source terms (2.2) with condition (2.3) allow model (2.1) to support a multiplicity of spatially uniform equilibria, i.e. real solutions $\left(\eta^{*}, \theta^{*}\right)$ of 


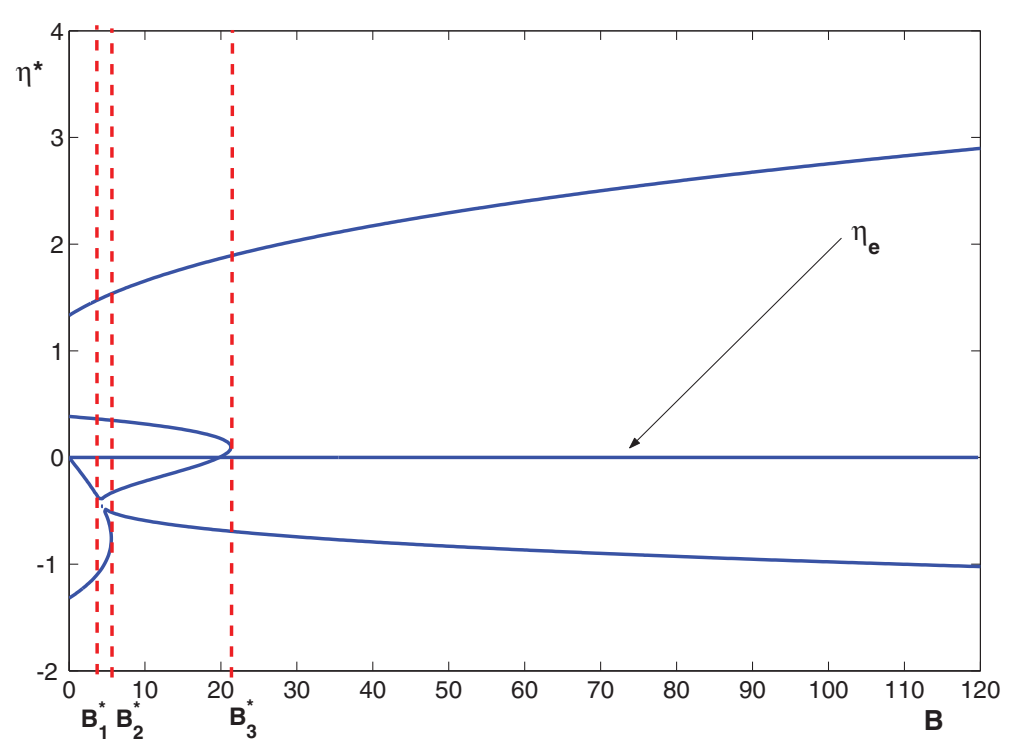

Figure 1. Bifurcation diagram in the $\left(B, \eta^{*}\right)$ plane showing how the number of spatially uniform equilibria $\left(\eta^{*}, \theta^{*}\right)$ for model (2.1)-(2.2) with assumption (2.3) changes upon varying the parameter $B$. The values for the other parameters are: $\alpha=0.5 ; \gamma=0.2 ; k_{2}=2.5 ; k_{3}=1.5 ; A_{1}=10 ; A_{2}=30$. The parameter $C$ has no role in deciding the number and the numerical value of spatially uniform equilibria. Here $\theta^{*}=0.5\left(20 \eta^{*}-60 \eta^{* 3}+B\right) /\left(10 \eta^{*}+B\right)$. In this specific case, three threshold values $B_{i}^{*}$ exist and there are three possible scenarios: 5 equilibria exist for $B \in\left(0, B_{1}^{*}\right) \cup\left(B_{2}^{*}, B_{3}^{*}\right) ; 7$ equilibria exist for $B \in\left(B_{1}^{*}, B_{2}^{*}\right) ; 3$ equilibria exist for $B>B_{3}^{*}$. We observe that $P_{e}=\left(\eta_{e}, \theta_{e}\right)=(0, \alpha)$ is always a spatially uniform equilibrium for the system.

the system

$$
\begin{aligned}
& A_{1}(1-\theta) \eta-A_{2} \eta^{3}-B(\theta-\alpha)=0 \\
& C\left(1+k_{2} \eta\right)(1-\theta)[1-\gamma(1-\theta)]-D \theta(1+\gamma \theta)-D k_{3} \eta \theta(1+\gamma \theta)=0 .
\end{aligned}
$$

As an example, in Figure 1 we show, for a specific choice of the other parameter values given in the figure caption, a bifurcation diagram in the $\left(B, \eta^{*}\right)$ plane showing how the number of spatially uniform equilibria $\left(\eta^{*}, \theta^{*}\right)$ changes by varying the parameter $B$.

Condition (2.3) ensures that: (i) the parameter $C$ has no role in deciding the number and the numerical value of spatially uniform equilibria; (ii) $P_{e}=\left(\eta_{e}, \theta_{e}\right)=(0, \alpha)$ is a spatially independent equilibrium for any choice of all parameter values.

Since $P_{e}$ is characterized by $\eta_{e}=0$, it corresponds to a flat electrode surface, from which corrugation and morphology can develop. For this reason, the related stability properties and possible destabilization mechanisms are particularly relevant from the physical point of view. Thus, in the following, we focus on $P_{e}$ and investigate the mechanisms responsible for pattern formation in (2.1)-(2.2). Toward this aim, we start by giving a brief overview on the $P_{e}$ destabilization mechanisms in the spatially uniform case. 


\section{The spatially uniform case: a brief overview}

The Jacobian matrix $J(\eta, \theta)$ evaluated at the homogeneous steady state $P_{e}$ is given by

$$
J\left(\eta_{e}, \theta_{e}\right)=\left[\begin{array}{cc}
A_{1}(1-\alpha) & -B \\
C\left(k_{2}-k_{3}\right) F_{1}(\alpha, \gamma) & -C F_{2}(\alpha, \gamma)
\end{array}\right]
$$

with

$$
F_{1}(\alpha, \gamma)=(1-\alpha)(1-\gamma+\alpha \gamma) ; \quad F_{2}(\alpha, \gamma)=\frac{2 \alpha \gamma(1+\alpha \gamma-\gamma)+1-\gamma}{\alpha(1+\alpha \gamma)} .
$$

Linear stability analysis easily shows that there are two specific bifurcations by which $P_{e}$ can lose its stability in the spatially uniform case. One is the transcritical bifurcation, where the attracting equilibrium $P_{e}$ loses its stability because it exchanges its stability properties with another equilibrium. Let be $\tau_{e}=\operatorname{tr}\left(J\left(\eta_{e}, \theta_{e}\right)\right)$ and $\delta_{e}=\operatorname{det}\left(J\left(\eta_{e}, \theta_{e}\right)\right)$. At the bifurcation value, $P_{e}$ becomes non-hyperbolic since one real eigenvalue of the related Jacobian matrix vanishes. Hence, the transcritical bifurcation can be detected by requiring $\delta_{e}=0$, i.e.

$$
B=\frac{A_{1}(1-\alpha) F_{2}(\alpha, \gamma)}{\left(k_{2}-k_{3}\right) F_{1}(\alpha, \gamma)}
$$

The other bifurcation involved is the Hopf one, where the attracting equilibrium $P_{e}$ loses its stability because a couple of complex conjugate eigenvalues of $J\left(\eta_{e}, \theta_{e}\right)$ crosses the imaginary axis. The Hopf bifurcation can then be detected by requiring $\tau_{e}=0$ and $\delta_{e}>0$, that is

$$
C=\frac{A_{1}(1-\alpha)}{F_{2}(\alpha, \gamma)}, \quad B>\frac{A_{1}(1-\alpha) F_{2}(\alpha, \gamma)}{\left(k_{2}-k_{3}\right) F_{1}(\alpha, \gamma)} .
$$

Figure 2(a) shows such bifurcation lines in the parameter space $(C, B)$ for a specific choice of the other parameter values: the vertical and horizontal ones are the Hopf and the transcritical lines, respectively. The regions on the right-hand side of the Hopf line and above the transcritical line are characterized by $\tau_{e}<0$ and $\delta_{e}>0$, so that, in the spatially uniform case, the homogeneous equilibrium $P_{e}$ is unconditionally stable.

For parameter choices below the transcritical bifurcation line, $\delta_{e}<0$ holds, so that $P_{e}$ can be destabilized by small homogeneous perturbations and the system trajectories tend toward a different stable steady state. Moreover, in the region on the left of the Hopf line and above the transcritical one, $P_{e}$ is unstable and we expect homogeneous oscillations due to the presence of a stable limit cycle, caused by a supercritical Hopf bifurcation. We will consider this scenario in more detail in Section 6. In the following, we focus on the spatial case and start by looking for the occurrence of the Turing instability phenomenon which - since Turing's pioneering intuition of 1952, [66] - has been considered one of the leading mechanisms for spatial pattern formation in reaction-diffusion systems.

\section{Pattern formation via Turing instability}

Using linear stability analysis, we show the occurrence of spatial patterns induced by diffusion, i.e. the diffusion-driven or Turing instability phenomenon for system (2.1)-(2.2). As widely known in literature, a reaction-diffusion system is said to exhibit diffusion-driven instability if a spatially uniform steady state - that is locally stable in the absence of 


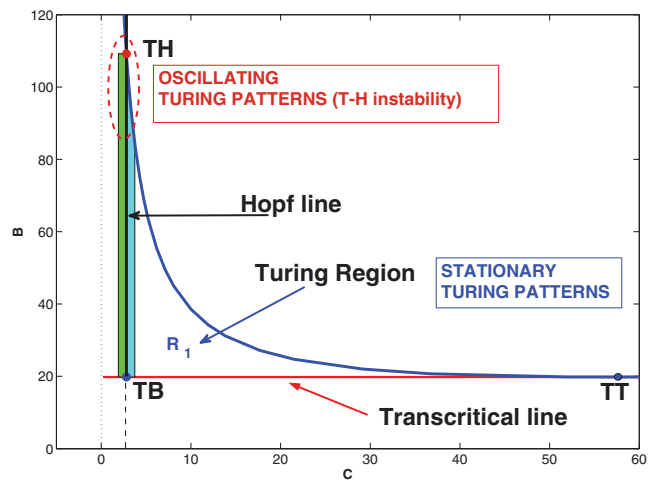

(a)

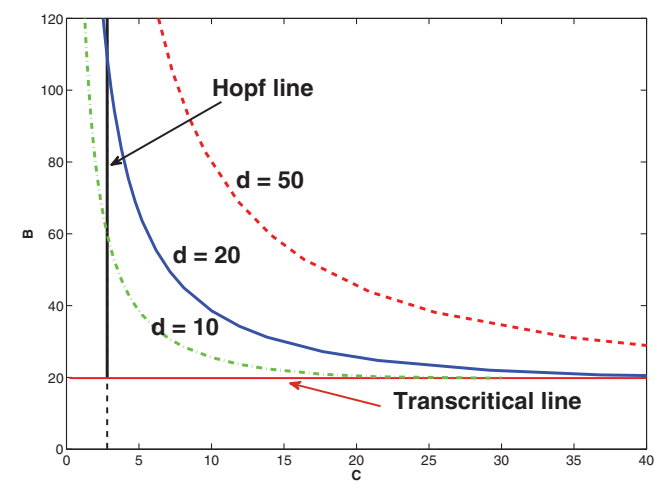

(b)

FIGURE 2. Bifurcation diagram in the parameter space $(C, B)$. The values for the other parameters are: $\alpha=0.5 ; \gamma=0.2 ; k_{2}=2.5 ; k_{3}=1.5 ; A_{1}=10 ; A_{2}=30$. (a) Detail of the bifurcation diagram for $d=20 . R_{1}$ is the region in the parameter space $(C, B)$ where conditions (4.4) for the spatial pattern initiation are satisfied. For this choice of the parameter values, the codimension-two bifurcation points $T H$ and $T B$ have coordinates $\left(C_{T H}, B_{T H}\right)=(2.8061,109.13),\left(C_{T B}, B_{T B}\right)=(2.8061,19.7979)$ (b) Bifurcation diagram in the parameter space $(C, B)$ : it clearly shows how, for increasing values of the parameter $d$, the related Turing region becomes larger.

diffusion - becomes unstable to small spatial perturbations when diffusion is present [51]. As far as the diffusion-driven instability is concerned, linear stability analysis is considered a useful technique to obtain conditions - expressed in terms of the system parameters for the development of unstable behaviour and to determine the characteristic length of the resulting spatial pattern. In the following, we briefly recall such conditions. To this end, we rewrite the electrodeposition model (2.1)-(2.2) in the following general form:

$$
\frac{\partial w}{\partial t}=\widetilde{D} \Delta w+S(w)
$$

with zero-flux boundary conditions on a $2 \mathrm{D}$ domain. In (4.1)

$$
w=\left(\begin{array}{l}
\eta \\
\theta
\end{array}\right), \quad \widetilde{D}=\left(\begin{array}{ll}
1 & 0 \\
0 & d
\end{array}\right), \quad S=\left(\begin{array}{c}
f(\eta, \theta) \\
g(\eta, \theta)
\end{array}\right),
$$

i.e. $w$ contains the system variables, $\tilde{D}$ contains the diffusion coefficients and $S$ accounts for the reaction kinetics. The homogeneous equilibrium $P_{e}=w_{e}=\left(\eta_{e}, \theta_{e}\right)$ satisfies $S\left(w_{e}\right)=0$ and its stability can be analysed by studying the behaviour of the system when a small inhomogeneous perturbation $\delta w$ is introduced in the neighbourhood of $w_{e}$, i.e. $w=w_{e}+\delta w$. The perturbation $\delta w$ can be written in terms of its spectral decomposition given by

$$
\delta w(z, t)=\sum_{k} c_{k} e^{\sigma_{k} t} U_{k}(z)
$$

where the $U_{k}(z)$ are the spatial eigenfunctions associated with the spatial eigenvalues $\lambda_{k}$, with $k \in \mathbb{N}$, of the unbounded non-negative linear operator $-\Delta$ with zero-flux boundary conditions on $\partial \Omega$. The eigenvalues $\sigma=\sigma\left(\lambda_{k}\right)$ account for the temporal part and describe 
the growth rate of the perturbation; they are obtained by solving the second order algebraic equation

$$
\left|J\left(\eta_{e}, \theta_{e}\right)-\lambda_{k} \widetilde{D}-\sigma I\right|=0,
$$

where $J\left(\eta_{e}, \theta_{e}\right)$ is the Jacobian matrix evaluated at the steady state $P_{e}$ and $I$ is the $2 \times 2$ identity matrix. The occurrence of Turing instabilities for a given $\lambda_{k}$ corresponds to the fact that equation (4.2) admits a positive solution $\sigma$.

For the general system (4.1), it can be easily shown that the Turing space - consisting of parameters resulting in Turing instability - is bounded by the following inequalities:

$$
\begin{aligned}
& J_{11}^{e}+J_{22}^{e}<0, \\
& J_{11}^{e} J_{22}^{e}-J_{12}^{e} J_{21}^{e}>0, \\
& d J_{11}^{e}+J_{22}^{e}>0, \\
& \left(J_{22}^{e}+d J_{11}^{e}\right)^{2}>4 d \delta_{e} .
\end{aligned}
$$

Here, $J_{i j}^{e}$ stands for the $i j$ entry of the Jacobian matrix $J\left(P_{e}\right)$ evaluated at the equilibrium $P_{e}=\left(\eta_{e}, \theta_{e}\right)$. The first two inequalities are derived by stability considerations on the homogeneous equilibrium $P_{e}$ in the absence of diffusion, the others are obtained by considerations on the onset of instability when diffusion is introduced. We refer to [51] and references therein for the explicit derivation of (4.3).

Taking into account these general conditions, we turn to the electrodeposition model (2.1)-(2.2) and show that the spatially homogeneous equilibrium $P_{e}=(0, \alpha)$ can undergo diffusion-driven instability. The set of conditions (4.3) for diffusion-driven instability hence specializes as

$$
\begin{aligned}
& \frac{A_{1}(1-\alpha)}{F_{2}(\alpha, \gamma)}<C<\frac{d A_{1}(1-\alpha)}{F_{2}(\alpha, \gamma)} \\
& B>\frac{A_{1}(1-\alpha) F_{2}(\alpha, \gamma)}{\left(k_{2}-k_{3}\right) F_{1}(\alpha, \gamma)} \\
& B<\frac{d^{2} A_{1}^{2}(1-\alpha)^{2}+C F_{2}(\alpha, \gamma)\left[2 A_{1} d(1-\alpha)+C F_{2}(\alpha, \gamma)\right]}{4 d C\left(k_{2}-k_{3}\right) F_{1}(\alpha, \gamma)} .
\end{aligned}
$$

We observe that $d>1$ is required in order to satisfy the first condition in (4.4). The other conditions hold when

$$
\begin{aligned}
& \frac{A_{1}(1-\alpha) F_{2}(\alpha, \gamma)}{\left(k_{2}-k_{3}\right) F_{1}(\alpha, \gamma)}<\frac{d^{2} A_{1}^{2}(1-\alpha)^{2}+C F_{2}(\alpha, \gamma)\left[2 A_{1} d(1-\alpha)+C F_{2}(\alpha, \gamma)\right]}{4 d C\left(k_{2}-k_{3}\right) F_{1}(\alpha, \gamma)} \\
& \quad \Leftrightarrow\left[d A_{1}(1-\alpha)-C F_{2}(\alpha, \gamma)\right]^{2}>0,
\end{aligned}
$$

that is always satisfied for $d A_{1}(1-\alpha) \neq C F_{2}(\alpha, \gamma)$. Inequalities (4.4) allow us to locate a region in the parameter space such that $P_{e}$ is stable to small perturbations in the absence of diffusion, but it can be unstable to small spatial perturbations when the diffusion is not neglected.

When diffusion mechanisms are considered, $P_{e}$ can destabilize in the parameter region where conditions for Turing instability are satisfied: the formation of stationary spatial 
patterns is hence expected because of the interaction between the non-linear reaction terms and the diffusion process. Figure 2(a) shows the Turing region $R_{1}$ in the parameter space $(C, B)$ for a fixed diffusion parameter $d=20$; Figure 2(b) shows how the Turing region $R_{1}$ becomes larger for increasing values of the diffusion parameter $d$.

At this point, it becomes particularly intriguing to investigate: (i) how the formation of inhomogeneous stationary patterns caused by Turing instabilities interacts with the appearance of homogeneous oscillations due to the Hopf bifurcation and (ii) if such interplay can lead to interesting classes of spatio-temporal patterns. The next sections are devoted to these topics.

\section{Higher-codimension bifurcation points}

The points where different bifurcation curves meet correspond to bifurcations of codimension higher than one which are likely to have a notable physical bearing since they entail the possibility of complex spatio-temporal dynamics. For example, the transcritical and the Turing bifurcation curves meet at the point $T T$ in Figure 2(a), whose coordinates are

$$
C_{T T}=\frac{d A_{1}(1-\alpha)}{F_{2}(\alpha, \gamma)}, \quad B_{T T}=\frac{A_{1}(1-\alpha) F_{2}(\alpha, \gamma)}{\left(k_{2}-k_{3}\right) F_{1}(\alpha, \gamma)},
$$

where $d>1$. Moreover, the two other points TB and TH in the bifurcation diagram in Figure 2(a) are interesting for the quest of potentially complex dynamics.

(i) Point $T B$, the intersection of the transcritical and Hopf lines, is characterized by the fact that both eigenvalues of the Jacobian matrix $J\left(\eta_{e}, \theta_{e}\right)$ are equal to zero. This indicates the occurrence of a Takens-Bogdanov bifurcation, in which a branch of Hopf bifurcations vanishes as the steady state of interest undergoes a saddle-node bifurcation. The occurrence of Takens-Bogdanov bifurcation is in many cases related to the existence of homoclinic bifurcations and is hence diagnostic of possible complex dynamics. In the parameter space $(C, B)$, the codimension-two bifurcation point TB has coordinates

$$
C_{T B}=\frac{A_{1}(1-\alpha)}{F_{2}(\alpha, \gamma)}, \quad B_{T B}=\frac{A_{1}(1-\alpha) F_{2}(\alpha, \gamma)}{\left(k_{2}-k_{3}\right) F_{1}(\alpha, \gamma)} .
$$

(ii) Point TH, where Hopf and Turing bifurcation curves meet, has coordinates

$$
C_{T H}=\frac{A_{1}(1-\alpha)}{F_{2}(\alpha, \gamma)}, \quad B_{T H}=\frac{A_{1}(1-\alpha) F_{2}(\alpha, \gamma)(1+d)^{2}}{4 d\left(k_{2}-k_{3}\right) F_{1}(\alpha, \gamma)} .
$$

In the neighbourhood of a TH point, the formation of inhomogeneous stationary patterns caused by Turing instabilities, interacts with the appearance of homogeneous oscillations due to a Hopf bifurcation possibly leading to the emergence of an interesting class of spatio-temporal patterns. TH instabilities in the neighbourhood of a $\mathrm{TH}$ point can in fact be considered an important mechanism for the appearance of spatio-temporal dynamics. 


\section{Turing-Hopf instabilities of the Hopf cycle}

The study of the TH instabilities of the spatially uniform periodic solution involves the interesting problem of understanding how the oscillations of the stable limit cycle can impact the formation of diffusive instabilities in a reaction-diffusion model. Inspired by [55], we investigate the formation of spatio-temporal patterns at the onset of TH instabilities. More precisely: (a) we consider the non-linear ODE reaction model and explicitly show the occurrence of a supercritical Hopf bifurcation by deriving an asymptotic expansion of the limit cycle solution $\Theta(t)$ which can develop after the Hopf bifurcation; (b) we investigate the onset of diffusive instabilities of the spatially uniform periodic solution for the electrodeposition model (2.1)-(2.2). To this end, we consider the model parameters in a neighbourhood of a codimension-two TH point, where the asymptotic expansion of the limit cycle and the Turing analysis of diffusive instabilities are both valid.

To address the above point (a), we first consider the reaction part of the electrodeposition model

$$
\begin{aligned}
& \dot{\eta}=f(\eta, \theta), \\
& \dot{\theta}=g(\eta, \theta),
\end{aligned}
$$

where $\dot{\eta}$ and $\dot{\theta}$ stand for the time derivatives of $\eta$ and $\theta ; f, g$ are given by (2.2) and (2.3). Recalling the analysis in the previous sections, we know that: (i) the spatially homogeneous equilibrium $P_{e}=\left(\eta_{e}, \theta_{e}\right)=(0, \alpha)$ is a stationary point for $(6.1)$; (ii) the Jacobian matrix $J\left(P_{e}\right)$ is given by (3.1)-(3.2); (iii) the Hopf bifurcation can be obtained by setting $\tau_{e}=0$ and $\delta_{e}>0$, namely by requiring (3.3) to hold. It follows that $P_{e}$ is stable for $\tau_{e}<0$, it loses its stability when $\tau_{e}=0$ and a limit cycle can arise surrounding the unstable steady state for $0<\tau_{e} \ll 1$.

In order to derive an asymptotic expansion of this limit cycle solution in the neighbourhood of the bifurcation point, we first translate the steady state $P_{e}$ onto the origin, by considering the new variables $U=\eta-\eta_{e}, V=\theta-\theta_{e}$ so that the model transforms to

$$
\dot{X}=J\left(P_{e}\right) X+\Psi(X),
$$

where $X=(U(t), V(t))$ and $\Psi(X)$ contains all non-linearities. Model (6.1), thus, becomes

$$
\begin{aligned}
\dot{U}= & A_{1} U(1-V-\alpha)-A_{2} U^{3}-B V, \\
\dot{V}= & C\left(1+k_{2} U\right)(1-V-\alpha)[1-\gamma(1-V-\alpha)] \\
& +C(V+\alpha)[1+\gamma(V+\alpha)]\left(1+k_{3} U\right) K(\alpha, \gamma),
\end{aligned}
$$

where $K(\alpha, \gamma)=\frac{(\alpha-1)(1-\gamma+\gamma \alpha)}{\alpha(1+\gamma \alpha)}$. In the following, we consider $B$ and $C$ as bifurcation parameters and we set the other parameter values to: $\alpha=0.5, \gamma=0.2, k_{2}=2.5, k_{3}=1.5$, $A_{2}=30, d=20, A_{1}=10$, so that we can refer to the bifurcation diagram in Figure 2(a). It is worth noting that parameters $\mathrm{B}, \mathrm{C}$ in model (1)-(2) are the coefficients for the processes related to adsorption, i.e. attachment of molecules to the growing surface. Accordingly one has

$$
\begin{aligned}
& \dot{U}=10(0.5-V) U-30 U^{3}-B V, \\
& \dot{V}=C(1+2.5 U)(0.5-V)(0.90+0.2 V)-0.8181 C(V+0.5)(1.10+0.2 V)(1+1.5 U) .
\end{aligned}
$$


Now we consider in (6.2) the invertible analytical change of coordinates

$$
Y=H(X)=\Gamma X+G(X)
$$

which transforms a neighbourhood of the origin into another one. If $H$ is such that

$$
Y=\left(\begin{array}{c}
z \\
\dot{z}
\end{array}\right),
$$

where $z(t)$ is an unknown function, then it is possible to show that the integration of system (6.1) can be reduced to the integration of a second order differential equation in the variable $z(t)$ [55]. Moreover, if $f$ and $g$ in (6.1) are polynomial functions of degree $M$, in the neighbourhood of the origin the transformation $H$ is close enough to the matrix transformation $\Gamma$ [55]. The main problem is how to choose the matrix transformation $\Gamma$. Theorem 6.1 addresses this question

Theorem 6.1 ( [55]) Let us assume that $\tau_{e}^{2}-4 \delta_{e}<0$. Then there exists an invertible transformation of variables in (6.4) such that (6.5) holds. The matrix $\Gamma$ is any non-trivial linear combination of

$$
\left\{\left(\begin{array}{cc}
1 & 0 \\
J_{11}^{e} & J_{12}^{e}
\end{array}\right), \quad\left(\begin{array}{cc}
0 & 1 \\
J_{21}^{e} & J_{22}^{e}
\end{array}\right)\right\} .
$$

The function $z$ in (6.5) satisfies the following second order equation:

$$
\ddot{z}-\tau_{e} \dot{z}+\delta_{e} z=G(z, \dot{z})
$$

where $G(z, \dot{z})$ does not involve linear terms in $z, \dot{z}$.

Remark 6.1 Following the proof of Theorem 6.1 in [55], we observe that the generators of $\Gamma$ can be obtained by solving the linear system

$$
J\left(\eta_{e}, \theta_{e}\right)\left(\begin{array}{l}
g_{11} \\
g_{12}
\end{array}\right)=\left(\begin{array}{l}
g_{21} \\
g_{22}
\end{array}\right),
$$

that is equivalent to define the matrix $\Gamma$ as a linear combination of the matrices in (6.6). Moreover, if $\Gamma$ satisfies (6.7), the following holds:

$$
\Gamma J\left(\eta_{e}, \theta_{e}\right) \Gamma^{-1}=\left(\begin{array}{cc}
0 & 1 \\
-\delta_{e} & \tau_{e}
\end{array}\right) .
$$

Let

$$
\Gamma=\left(\begin{array}{ll}
g_{11} & g_{12} \\
g_{21} & g_{22}
\end{array}\right) .
$$

For the chosen parameter values, one has $\tau_{e}=5-1.7818 C$ and $\delta_{e}=C(0.45 B-8.9090)$. 
Then by (6.8) it follows, through straightforward algebra,

$$
\Gamma \propto\left(\begin{array}{cc}
0.2 & 0 \\
1 & -0.2 B
\end{array}\right) .
$$

Accordingly, we consider the new variable

$$
z=0.2 U, \quad \dot{z}=U-0.2 B V+o\left(U V, U^{2}, V^{2}\right),
$$

so that system (6.3) is equivalent to the second order equation for the unknown $z$

$$
\ddot{z}-\tau_{e} \dot{z}+\delta_{e} z=G(z, \dot{z})
$$

where

$$
\begin{aligned}
G(z, \dot{z})= & 250 \frac{z \dot{z}}{B}-1250 \frac{z^{2}}{B}-3750 z^{3}-9 \times 10^{-12} B C+1.8181 \frac{C \dot{z}^{2}}{B}-18.1818 \frac{C z \dot{z}}{B} \\
& +45.4545 \frac{C z^{2}}{B}-17.3636 C z \dot{z}+86.8181 C z^{2}+18.6363 \frac{C z \dot{z}^{2}}{B} \\
& -186.3636 \frac{C z^{2} \dot{z}}{B}+465.9090 \frac{C z^{3}}{B} .
\end{aligned}
$$

Seeking an oscillation with small finite positive amplitude $\epsilon$, we set

$$
z(t)=\epsilon \zeta(t)
$$

obtaining from (6.9) the following weakly non-linear oscillator

$$
\ddot{\zeta}-\sigma \dot{\zeta}+\rho \zeta=\epsilon G_{1}(\zeta, \dot{\zeta}, \epsilon)
$$

with

$$
\begin{aligned}
G_{1}(\zeta, \dot{\zeta}, \epsilon)= & 250 \frac{\zeta \dot{\zeta}}{B}-1250 \frac{\zeta^{2}}{B}-3750 \epsilon \zeta^{3}-9 \times 10^{-12} B C \epsilon^{-2}+1.8181 \frac{C \dot{\zeta}^{2}}{B}-18.1818 \frac{C \zeta \dot{\zeta}}{B} \\
& +45.4545 \frac{C \zeta^{2}}{B}-17.3636 C \zeta \dot{\zeta}+86.8181 C \zeta^{2}+18.6363 \frac{\epsilon C \zeta \dot{\zeta}^{2}}{B} \\
& -186.3636 \frac{\epsilon C \zeta^{2} \dot{\zeta}}{B}+465.9090 \frac{\epsilon C z^{3}}{B} .
\end{aligned}
$$

It follows that the cyclic solution of system (6.1) corresponds to the non-trivial periodic solution of the second-order differential equation (6.10). To find an asymptotic expansion of such a solution, we apply the Krilov-Bogoliubov-Mitropolski averaging method $[4,62]$. We hence introduce the new variables $r=r(t)$ and $\beta=\beta(t)$ such that

$$
\zeta=r \cos (t+\beta) ; \quad \dot{\zeta}=-r \sin (t+\beta)
$$


The corresponding averaged equations are, therefore,

$$
\begin{aligned}
\dot{r} & =\frac{r}{2}\left[\tau_{e}-p(r, \epsilon)\right], \\
\dot{\beta} & =q(r, \epsilon),
\end{aligned}
$$

with $p(r, \epsilon)$ and $q(r, \epsilon)$ given by

$$
\begin{aligned}
& p(r, \epsilon)=\frac{\epsilon}{\pi r} \int_{0}^{2 \pi} \sin \Phi G_{1}(r \cos \Phi,-r \sin \Phi ; \epsilon) d \Phi \\
& q(r, \epsilon)=\frac{-\epsilon}{2 \pi r} \int_{0}^{2 \pi} \cos \Phi G_{1}(r \cos \Phi,-r \sin \Phi ; \epsilon) d \Phi .
\end{aligned}
$$

Direct integration yields

$$
p(r, \epsilon)=46.5909 \frac{\epsilon^{2} r^{2} C}{B}, \quad q(r, \epsilon)=\epsilon^{2} r^{2}\left[1406.25-177.04 \frac{C}{B}\right] .
$$

Taking $\epsilon^{2}=\left|\tau_{e}\right|$ as in [55], we obtain

$$
\dot{r}=\frac{r}{2} \tau_{e}\left[1-46.5909 r^{2} \frac{C}{B}\right], \quad \dot{\beta}=r^{2} \tau_{e}\left[1406.25-177.04 \frac{C}{B}\right] .
$$

If $\tau_{e}>0$, an orbitally asymptotically stable limit cycle exists with $r^{2}=0.0214 \frac{B}{C}$ and $\dot{\beta}=\tau_{e}\left[30.1829 \frac{B}{C}-3.8\right]$. We can then provide the uniform asymptotic expansion of the solution of (6.9) in terms of the small parameter $\tau_{e}$

$$
z(t)=\epsilon \zeta(t)=\epsilon \cos (t+\beta)=0.14628 \sqrt{\frac{\tau_{e} B}{C}} \cos \left[1+\tau_{e}\left(30.18 \frac{B}{C}-3.8\right)\right] t+o\left(\tau_{e}\right),
$$

and obtain the cycle solution $\Theta(t)=(\bar{\eta}(t), \bar{\theta}(t))$ of system $(6.1)$

$$
\bar{\eta}(t)=\eta_{e}+5 z(t), \quad \bar{\theta}(t)=\theta_{e}-5 B^{-1}[\dot{z}(t)-5 z(t)] .
$$

From the above representation, it clearly appears that for $\tau_{e}>0$ and close enough to the bifurcation value, i.e. $0<\tau_{e} \ll 1$, an orbitally asymptotically stable limit cycle appears for the system (6.1) that surrounds the unstable equilibrium $\left(\eta_{e}, \theta_{e}\right)$. For $\tau_{e} \rightarrow 0$, the limit cycle shrinks toward $\left(\eta_{e}, \theta_{e}\right)$ which changes stability crossing the line $\tau_{e}=0$. In other words, $\left(\eta_{e}, \theta_{e}\right)$ undergoes a supercritical Hopf bifurcation. Such a phenomenology is clearly depicted in Figure 3 where we show the numerical solutions of the ODE system (6.1) obtained by the Matlab routine ode45 for a selection of $C$ values near the Hopf bifurcation point.

We can now address point (b) by examining the ways in which the above limit cycle can give rise to Turing instabilities near a $\mathrm{TH}$ point. $\mathrm{TH}$ instabilities generated by the limit cycle take place when a temporal eigenvalue $\sigma$ - a root of equation (4.2) - exists such that $\operatorname{Re}(\sigma)>0$. Such TH instabilities are defined as weak if equation (4.2) has at least one real root $\sigma>0$ and strong if the roots $\sigma$ are complex-conjugate with positive 

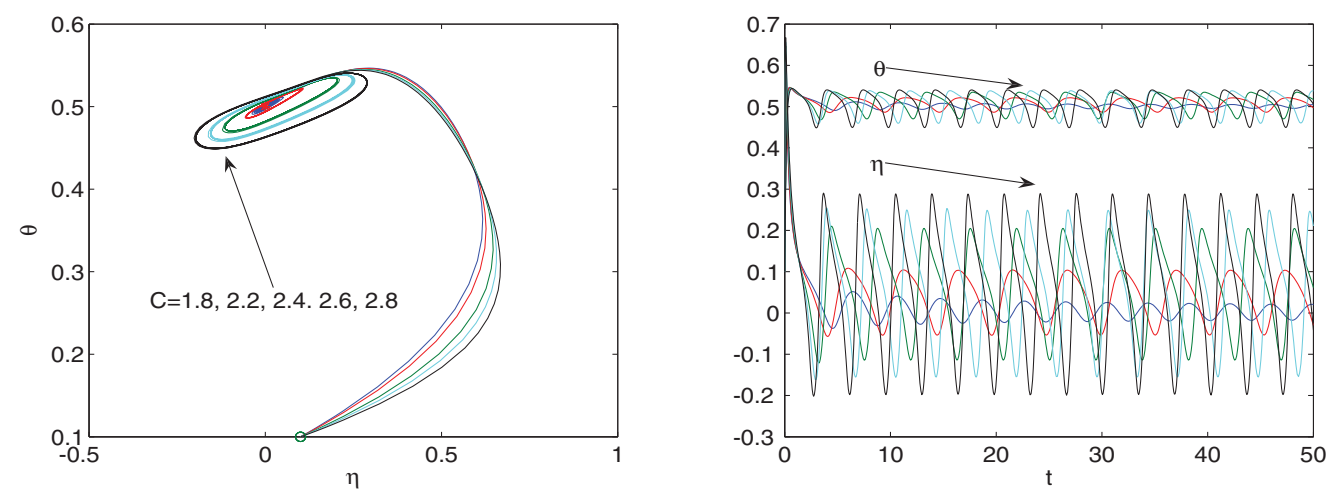

(a)
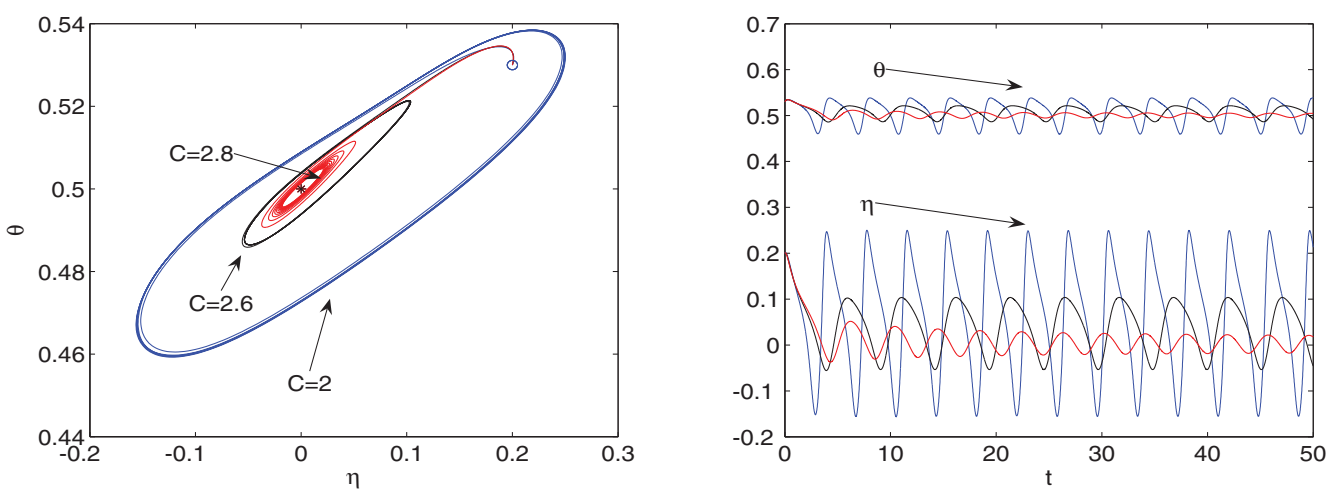

(b)

FIGURE 3. Occurrence of a supercritical Hopf bifurcation. Numerical solutions of the ODE system (6.1) for $B=22$ and $C$ chosen in the neighbourhood of the Hopf bifurcation value $C^{*}=2.8061$. By increasing $C$ up to $C^{*}$, progressively smaller limit cycles are obtained, collapsing on the equilibrium $P_{e}$.

real part. The following Theorem 6.2 states the conditions for the occurrence of weak or strong TH instabilities.

Theorem 6.2 ([55]) Let $\lambda_{k}$ be a positive spatial eigenvalue. Let $\tau_{T}$ and $\delta_{T}$ be defined as

$$
\tau_{T}=\operatorname{tr}\left(J\left(\eta_{e}, \theta_{e}\right)-\lambda_{k} \tilde{D}\right), \quad \delta_{T}=\operatorname{det}\left(J\left(\eta_{e}, \theta_{e}\right)-\lambda_{k} \tilde{D}\right) .
$$

Further assume that the reaction system has a limit cycle attained via a Hopf bifurcation. If $\tau_{T} \leqslant 0$ and $\delta_{T}<0$ then weak TH instabilities appear. If $\tau_{T}>0$, TH instabilities appear and they are weak provided $\tau_{T}^{2}-4 \delta_{T} \geqslant 0$ while they are strong if $\tau_{T}^{2}-4 \delta_{T}<0$.

As far as the features of the expected patterns are concerned, weak instabilities are characterized by dominant inhomogeneous steady patterns onto which slightly time periodic oscillations can be overlapped, exhibiting the same frequency as the limit cycle. 
Strong instabilities instead feature intermittent switching between: (i) the inhomogeneous patterns, represented by the set of positiveness of the spatial eigenfunction, and (ii) its complementary pattern represented by the set of negativeness of the spatial eigenfunction. In such a case $\sigma_{i}=\sqrt{\delta_{T}-\tau_{T}^{2} / 4}$ is the frequency of oscillations of the pattern that is different from the frequency of the limit cycle [55].

\section{Numerical investigations}

Hexagonally arranged spots or stripes are typical Turing patterns in 2D reaction-diffusion systems, but also rhombic or labyrinthine patterns have been observed [35]. In order to accurately predict the spatial features of the expected Turing patterns, non-linear bifurcation analysis and the amplitude equations formalism must be used, see i.e. [19,22, $29,30]$. Nevertheless, this kind of analysis is beyond the scope of the present paper, for this reason we resorted to numerical investigation of pattern selection issues.

In the following, we employ the same set of parameter values adopted for Figure 2(a) and we perform a numerical study to support the analytical results obtained in the previous sections. We recall that we consider $B$ and $C$ as the only bifurcation parameters and that the Turing Region in the parameter space $(C, B)$ is the region $R_{1}$ indicated in Figure 2(a). For this choice of the parameter values, the Hopf threshold is $C=C_{\text {hopf }}=2.8061$ with $B>19.7979$ and the codimension-two bifurcation points $T H$ and $T B$ have coordinates $\left(C_{T H}, B_{T H}\right)=(2.8061,109.13),\left(C_{T B}, B_{T B}\right)=(2.8061,19.7979)$, respectively. We show that the system can exhibit patterns both inside and outside the Turing region and that the features of the obtained patterns can be very different, as a result of the specific destabilization mechanism under consideration.

In order to solve the reaction-diffusion PDE system on the 2D spatial domain $\left[0, L_{x}\right] \times\left[0, L_{y}\right]$ and for $t \in[0, T]$ we apply the Extended Central Difference Formulas (ECDF) for semi-discretization in space coupled with the ADI method in time that approximate implicitly the diffusion term and explicitly the reaction terms. ADI-ECDF schemes of order $p=2,4,6$ have been recently introduced in [63] to deal with the approximation of Turing patterns that are stationary solutions of the PDE system in an accurate and efficient way with a flexible choice of integration stepsizes both in space and time. This task is challenging from the computational point of view because high accuracy is required both in space, to capture the pattern structure, and in time, to attain the stationary pattern in a stable way. Moreover, in [64] this approach has been successfully applied to deal with the case of oscillating Turing patterns arising from the superimposition of an external forcing term in the source, representing sinusoidally modulated electrochemical control resulting in a notable smoothing effect. For these reasons, we decided to apply the ADI-ECDF scheme of order $p=4$ in space, as a good compromise between accuracy and computational cost. Hence, we approximate the model introduced in this paper for sets of parameters corresponding to Turing and $\mathrm{TH}$ regions, where stationary patterns and patterns oscillating in space and time are expected, respectively.

In Figures 4-6, we show (right panels) two auxiliary quantities that allow a more straightforward presentation of our numerical results 

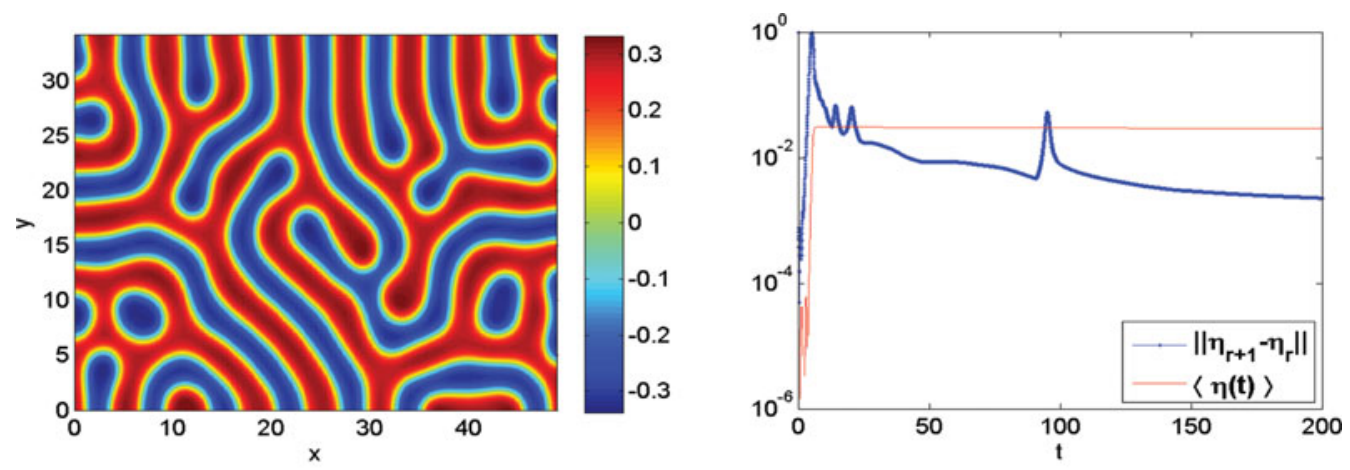

(a)
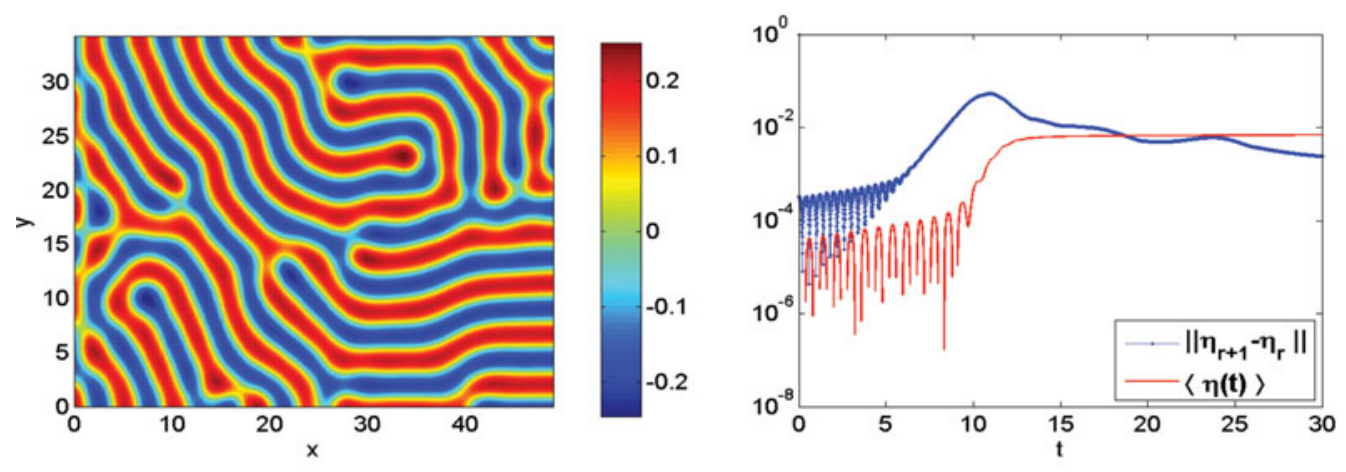

(b)

FIgURE 4. Spatial patterns in the Turing Region (a) $B=30, C=3, T_{f}=200$ (b) $B=66, C=3$, $T_{f}=30$.

(i) $\left\|\eta_{r+1}-\eta_{r}\right\|_{2}$ : the difference in norm between two consecutive patterns, i.e. the numerical approximations at the times $t_{r+1}$ and $t_{r}$ along the time integration. This quantity quantifies the tendency of the solution to reach a stationary pattern: in this case this difference should tend to zero (we can stop the time integration when this value is less than an appropriate threshold);

(ii) $\langle\eta(t)\rangle$ : an approximation of the integral $\frac{1}{|\Omega|} \int_{\Omega} \eta(x, y, t) d x d y$. This second indicator is a spatial mean value that, for longtime integration, allows us to discriminate between stationary and oscillating patterns. In fact, if $\langle\eta(t)\rangle$ reaches an approximately constant value, a stationary pattern tends to be attained, while an asymptotic oscillatory behaviour of $\langle\eta(t)\rangle$ indicates an oscillating pattern.

It is worth stressing that, to distinguish between weak and strong $\mathrm{TH}$ instabilities these two indicators are not sufficient. For this reason, in these cases, a selection of snapshots of the numerical solutions at different integration times will be presented.

We first address the case of spatial pattern formation via Turing instability.

We consider parameter values in the Turing Region $R_{1}$ in Figure 2(a) and take as initial conditions random spatial perturbations of the stable homogeneous steady state $P_{e}$. For our simulations, we consider the rectangular domain $\Omega=[0,50] \times[0,36]$ and 

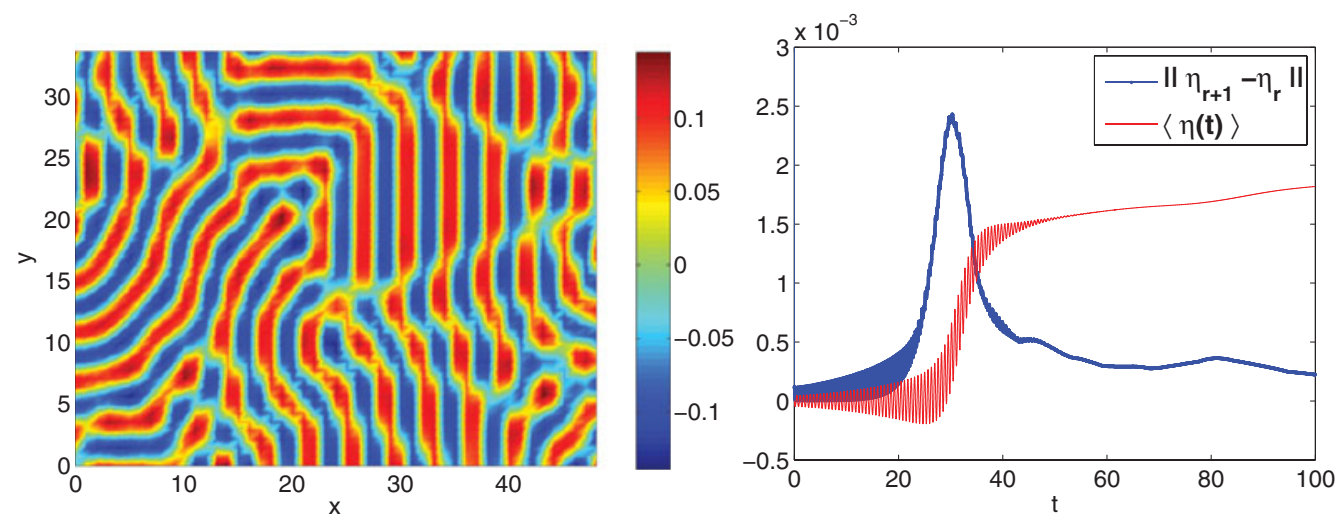

(a)
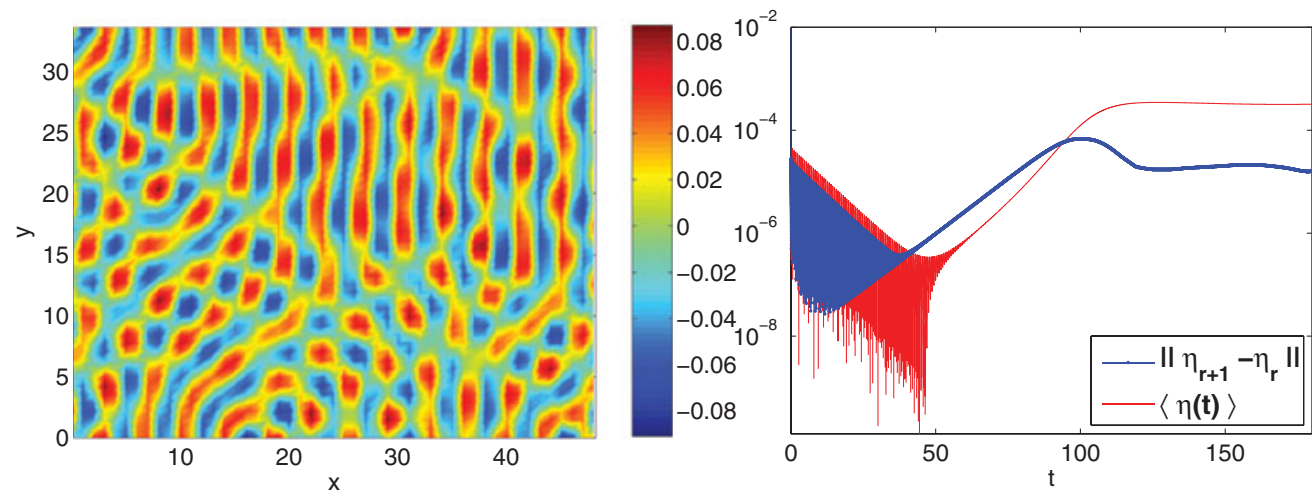

(b)

Figure 5. Spatial patterns for the morphology $\eta(x, y, t)$ in the Turing Region. (a) $B=90, C=3, T_{f}=100$ (b) $B=99, C=3, T_{f}=180$.

$N_{x}=N_{y}=50$ space mesh points. For time integration we apply the ADI-ECDF method with $h_{t}=0.001$. The final time is indicated as $T_{f}$.

Figures 4-5 show the Turing patterns of the morphology $\eta$ in different points of the Turing Region $R_{1}$, i.e. for different $(C, B)$ values, to emphasize the role of the parameters $B$ and $C$ in the resulting pattern typology. In order to better elucidate this aspect, we start from a point well inside the Turing region $R_{1}$, i.e. $B=30$ and $C=5$, and then move across $R_{1}$ towards the point $T H$ along the line $C=3$. Figure 4(a), corresponding to the pattern developed well inside the Turing region $R_{1}$, depicts a pseudo-labyrinth stationary Turing pattern. By increasing the value of $B$ and moving toward the Hopf line, i.e. by considering $B=66$ and $C=3$, such pattern turns into the more connected labyrinthinelike structure depicted in Figure 4(b). This qualitative trend is well confirmed by further increasing the value of $B$ along the $C=3$ line, as shown by the simulation in Figure 5(a) for $B=90$. Moreover, inside $R_{1}$, the pattern typology changes again when approaching the neighbourhood of the point $T H$ : in fact, when $B=99$, Figure 5(b) shows how the labyrinthine-like structure breaks down yielding a stationary tessellation pattern. In the 

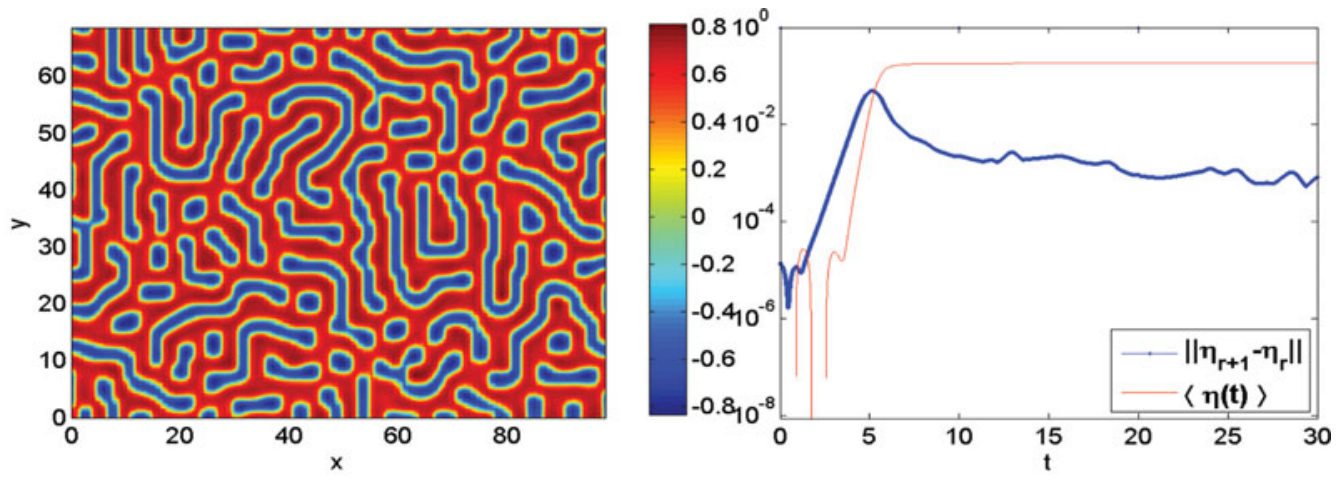

(a)
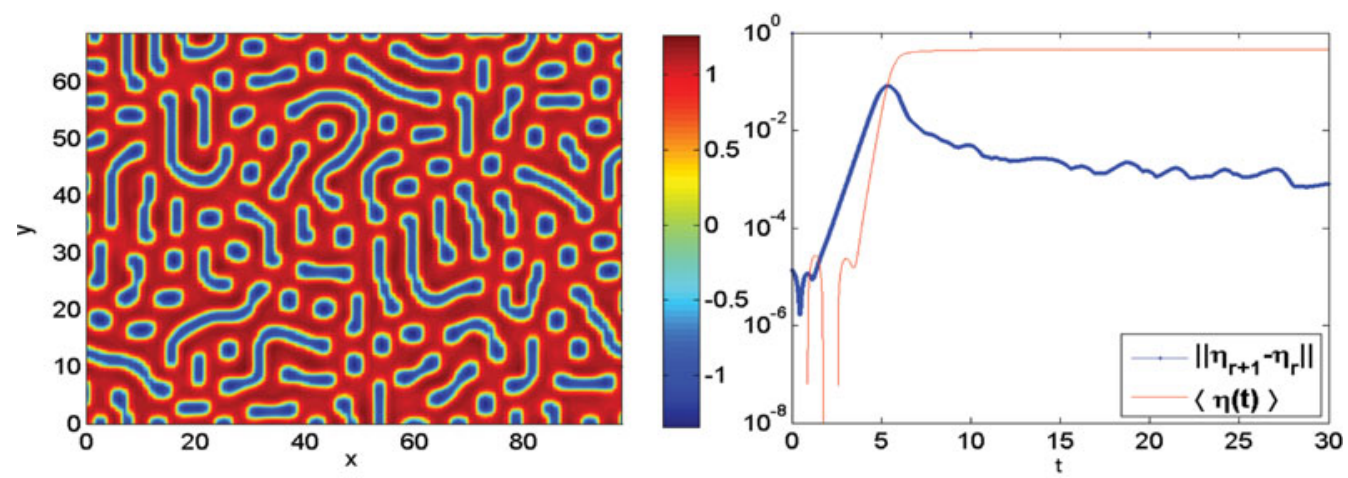

(b)

FIgURE 6. Spatial patterns in the Turing Region $R_{1}$ obtained by varying the parameter $A_{2}$. Here $\Omega=[0,100] \times[0,70], d=20, B=30, C=3$. (a) $A_{2}=5$ (b) $A_{2}=2$.

right panels of Figures $4-6$, the corresponding increments and the time integrals $\langle\eta(t)\rangle$ show that stationary patterns are obtained at the final time of integration $T_{f}$.

It is worth stressing the peculiar role of the parameter $A_{2}$ on the features of the Turing patterns. From the arguments highlighted in Sections 3-4, the bifurcation thresholds do not depend on $A_{2}$ and then it is possible to conclude that $A_{2}$ has no impact on the destabilization of the homogeneous equilibrium $P_{e}$ through the transcritical or the Hopf bifurcation in the spatially uniform case or through the Turing bifurcation in the spatial case. Hence, by varying only $A_{2}$, the two parameter bifurcation diagram in Figure 2(a) remains unchanged. Neverthless, numerical investigations show that $A_{2}$ has an interesting role in pattern selection.

We found that, well inside the Turing Region $R_{1}$ (e.g. for low values of $B$ within $R_{1}$ ), low values of $A_{2}$ cause a disruption of the labyrinthine structure, resulting in reversed spots and worms Turing patterns. The qualification reversed indicates that such spots and worms actually represent holes in the morphology, rather than outgrowth structures as in the conventional case. The more $A_{2}$ is decreased the more fragmented the spottyworms pattern appears, with high prevalence of reversed spotty structures, as shown in Figure 6(b). By fixing low values of $A_{2}$ and moving toward the $\mathrm{TH}$ point (e.g. for 

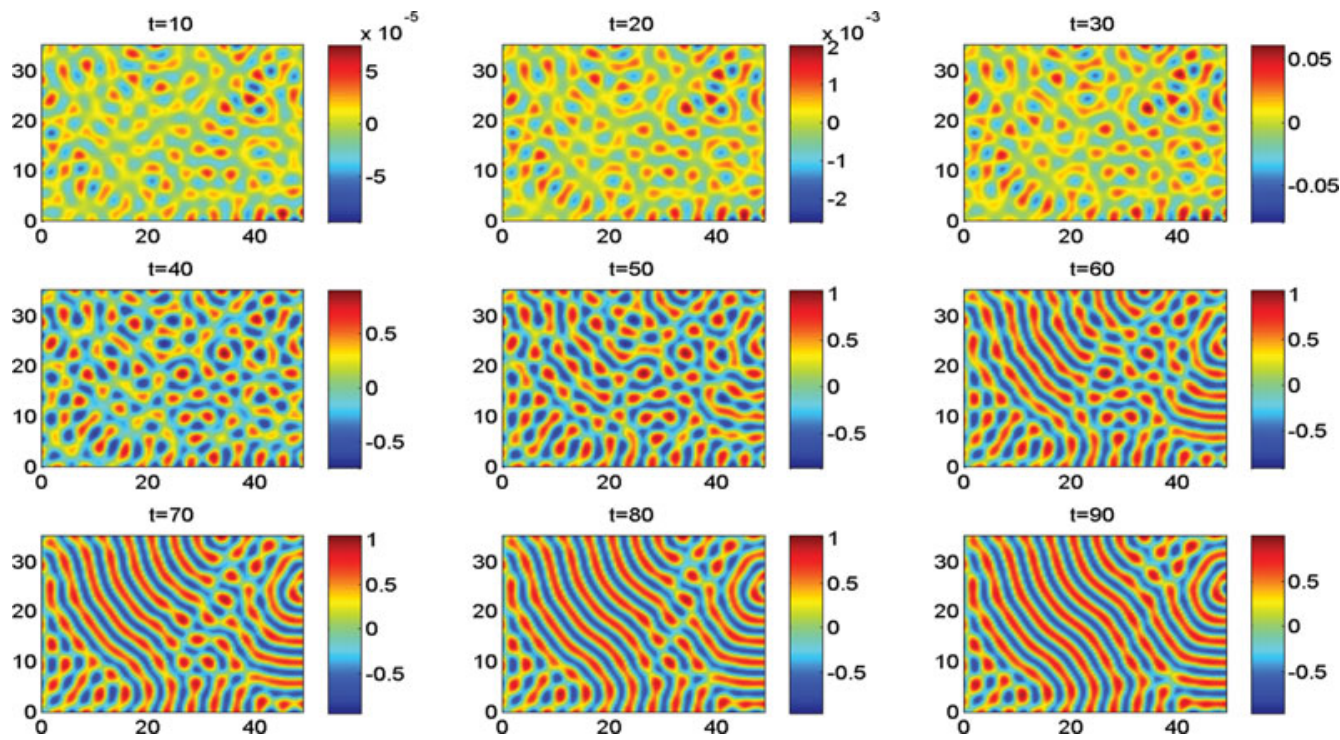

Figure 7. Case $d=20, B=90, C=3$. Numerical simulations for $A_{2}=1$. Snapshots show different stages of the spatial pattern formation at times $t=10,20, \ldots, 90$.

higher values of $B$ ), in the transient dynamics one observes spotty structures while in the stationary limit an intriguing coexistence is found between spots and stripes, as showed in Figures 7 and 8.

The physico-chemical basis of the special role of $A_{2}$, as evident from equation (2.2) and as detailed in [15], resides in the fact that it defines the rate of metal deposition far from equilibrium. This parameter can be easily tuned in an electrodeposition experiment, e.g. by changing the type of ligand in the electroactive metal complex or by properly adjusting the potentiostatic electrodeposition conditions (the latter approach is easier from the operational point of view, but requires an insightful understanding of the relevant electrokinetics and respective mathematical modelling). Moreover, $B$ quantifies the impact of the adsorbate on metal growth. In Figure 9 a selection of electrodeposits is shown exhibiting conventional and reversed spots and worms obtained by varying $A_{2}$ and $B$. The experiments refer to $\mathrm{Au}$ and $\mathrm{AuMn}$ electrodeposition from a cyanide bath based on a deep eutectic solvent: on the basis of in situ spectroscopic evidence [16,17], cyanide can be regarded as the species corresponding to $\theta$. Au and AuMn exhibit lower and higher surface interactions with cyanide: the former metal can, thus, be regarded as a case with lower $B$ than the second one. In our example, $A_{2}$ is controlled by adjusting the applied electrodic potential. It can be noticed that: low $A_{2}$ and $B$ (Panel A) corresponds to reversed spots and worms; high $A_{2}$ while low $B$ (Panel B) corresponds to conventional spots and worms.

\subsection{Spatio-temporal dynamics near the Hopf line}

In this subsection, we numerically investigate the spatio-temporal system dynamics moving toward the Turing Region from the Hopf one. We choose the parameter values near the 

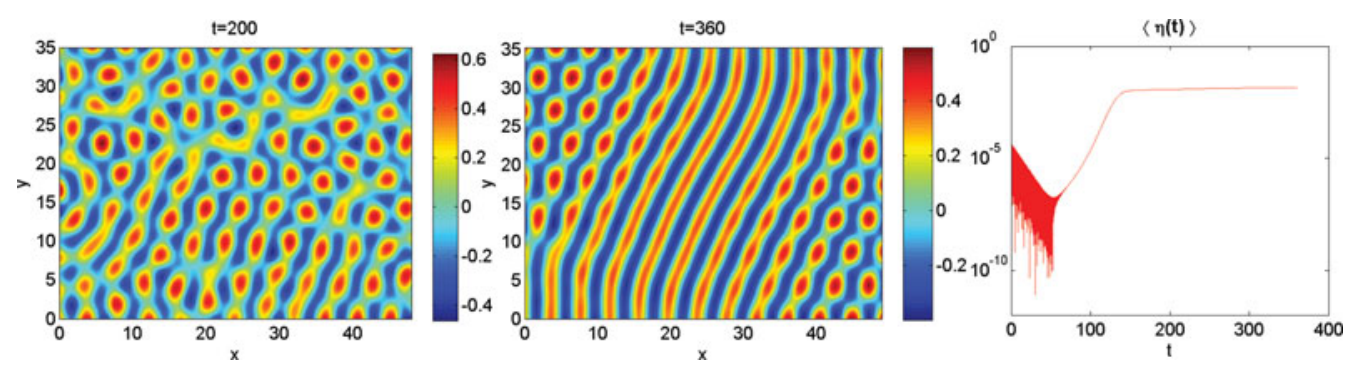

Figure 8. Case $d=20, B=99, C=3, A_{2}=1$. Snapshots of the spatial patterns in the Turing Region $R_{1}$ at two different times: $t=200$ (left panel), $t=360$ (middle panel). In the right hand-side panel, the time dependent behaviour of $\langle\eta(t)\rangle$ shows that at $T_{f}=360$ the pattern has reached its asymptotic state.

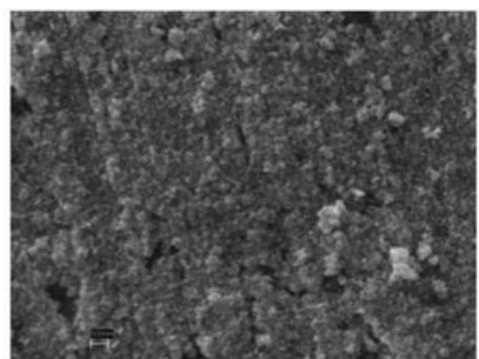

(A)

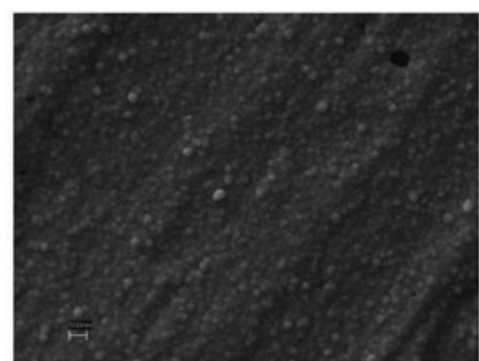

(B)

Figure 9. $\mathrm{Au}(\mathrm{A})$ and AuMn (B) experimental electrodeposits obtained at $-1.1 \mathrm{~V}$ versus $\mathrm{Au}$ quasi-reference electrode from $\mathrm{Au}(\mathrm{I})$ cyanocomplexes baths without and with $\mathrm{MnCl}_{2}$ employing a deep eutectic solvent as the electrolyte. See the discussion in the main text.

Hopf bifurcation line, that is $B=109, C=2.794$. In Figures $10-12$, a fascinating competition between the Hopf and Turing instabilities is shown. As discussed in the following, the winner of the space vs time competition is decided by the value of the diffusion parameter $d$.

In Figure 10, we show the results of a representative numerical simulation carried out until $T_{f}=200$, with the diffusion parameter $d=20$. Figure 10(a) shows some snapshots of the morphology $\eta(x, y, t)$ at different time values during the evolution: in the transient period spotty patterns and tessellation can be recognized, while a spatially homogeneous pattern oscillating in time is attained asymptotically for $t \geqslant 150$. In Figure 10(b), left panel, we show the dynamics of the spatial average $\langle\eta(t)\rangle$, the inset highlights its oscillating behaviour. In the right panel, we show the corresponding limit cycle for $(\langle\eta\rangle,\langle\theta\rangle)$. The initial condition $\left(\eta_{0}, \theta_{0}\right)$ is a spatially random perturbation of the homogeneous equilibrium $\left(\eta_{e}, \theta_{e}\right)=(0,0.5)$ and in fact the limit cycle is attained starting from inside.

Figures 11 and 12 show how the above spatio-temporal behaviour completely changes by increasing the value of the diffusion coefficient $d$. For values of $d$ higher that $d=20$, in the competition the spatial structure survives with respect to the homogeneous temporal oscillations. Hence, in this case, stationary spatial patterns are present also in the Hopf region, but only in a close neighbourhood of the Turing region. In Figure 11(a) the case $d=100$ is presented: the selection of time snapshots shows that - after a transient 

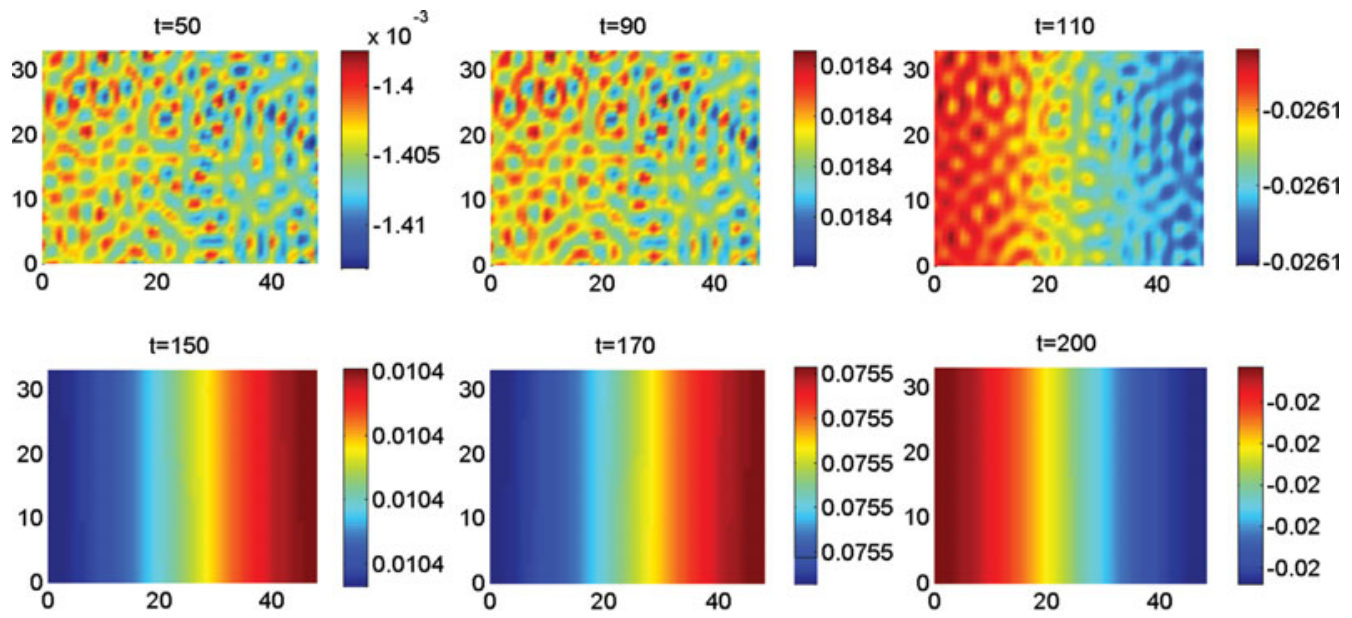

(a)
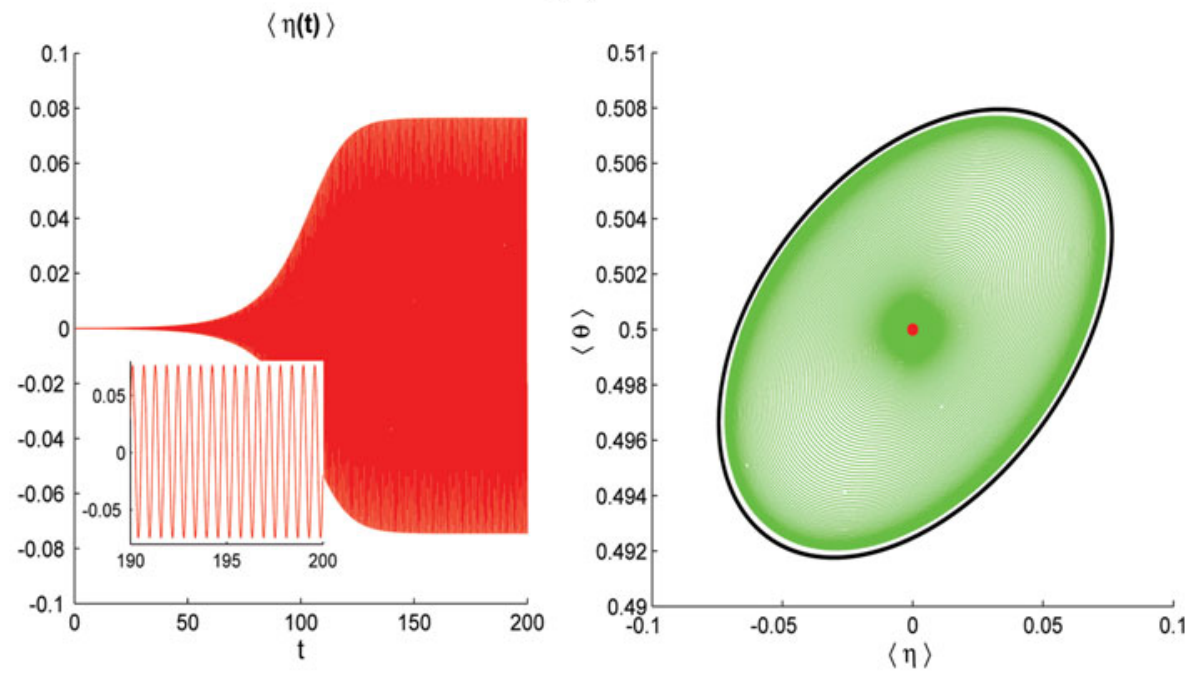

(b)

FIGURE 10. Spatio-temporal dynamics in the Hopf region, near the Hopf bifurcation line in the case $d=20$. The numerical values for the bifurcation parameters are: $B=109, C=2.794$. (a) Snapshots of $\eta(x, y, t)$ at different time values during evolution: a homogeneous pattern oscillating in time is attained for $t \geqslant 150$. (b) Left panel: spatially averaged value $\langle\eta\rangle$. Right panel: corresponding limit cycle in the phase plane $(\langle\eta\rangle,\langle\theta\rangle)$.

regime, where a spotty solution oscillating in time is present - an entangled labyrinthine pattern is attained as the stationary spatial pattern. The left panel of Figure 11(b) shows the dynamics of $\langle\eta(t)\rangle$ until $T_{f}=10$; in the right panel the corresponding limit cycle is shown. As expected, for very large values of $d$ the spatial structure tends to prevail. Moreover, in the case $d=22,735$, no qualitative changes appear in the obtained phenomenology as it can be observed in Figure 12. We recall that by increasing the value of the parameter 
$t=1$

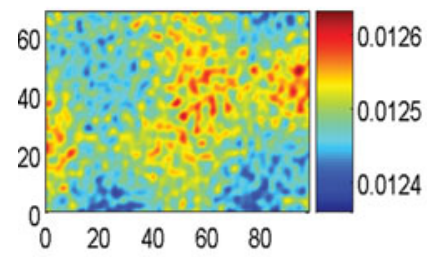

$t=4$

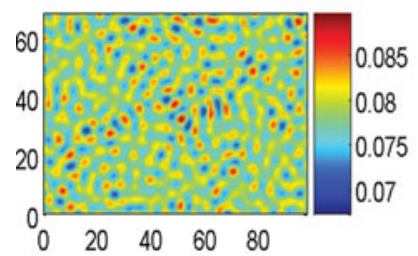

$t=7$

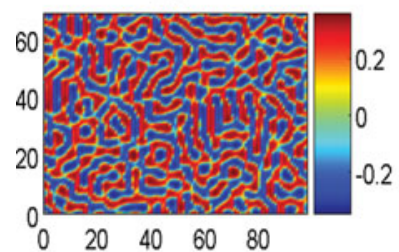

$t=2$

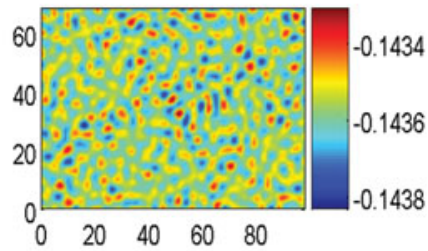

$t=5$

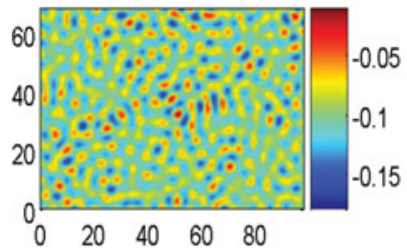

$t=8$

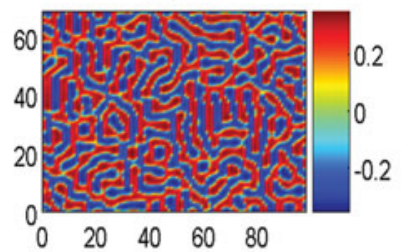

$t=3$

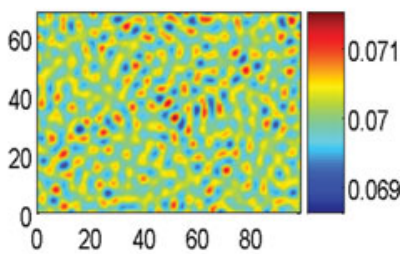

$t=6$

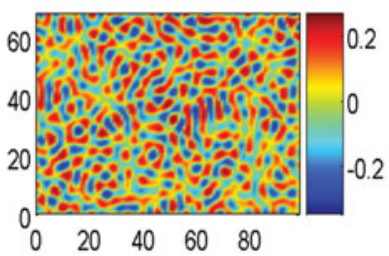

$t=9$

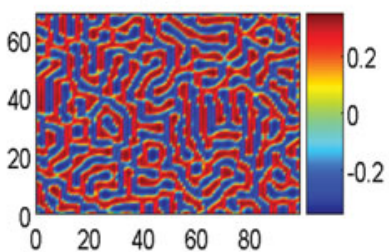

(a)
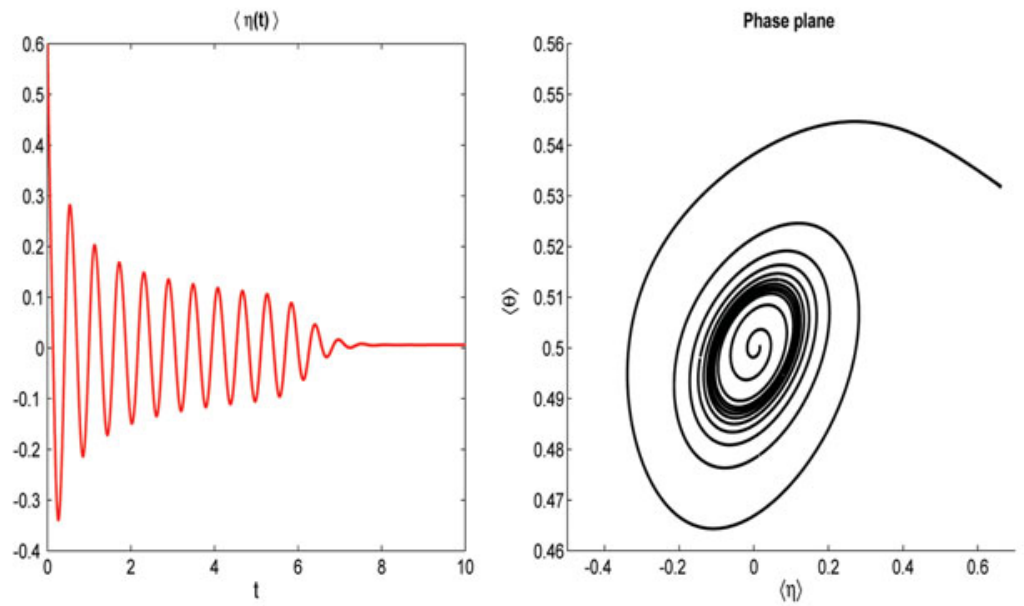

(b)

FiguRE 11. Spatio-temporal dynamics in the Hopf region, near the Hopf bifurcation line in the case $d=100$. The numerical values for the bifurcation parameters are: $B=109, C=2.794$. In this case the spatial domain is $\Omega=[0,100] \times[0,70]$. (a) Snapshots at times $t=1,2, \ldots, 9$ of the spatial patterns. (b) Left panel: spatially averaged value $\langle\eta(t)\rangle$ until $T_{f}=10$. Right panel: corresponding dynamics in the phase plane $(\langle\eta\rangle,\langle\theta\rangle)$, where a stable focus is attained. 

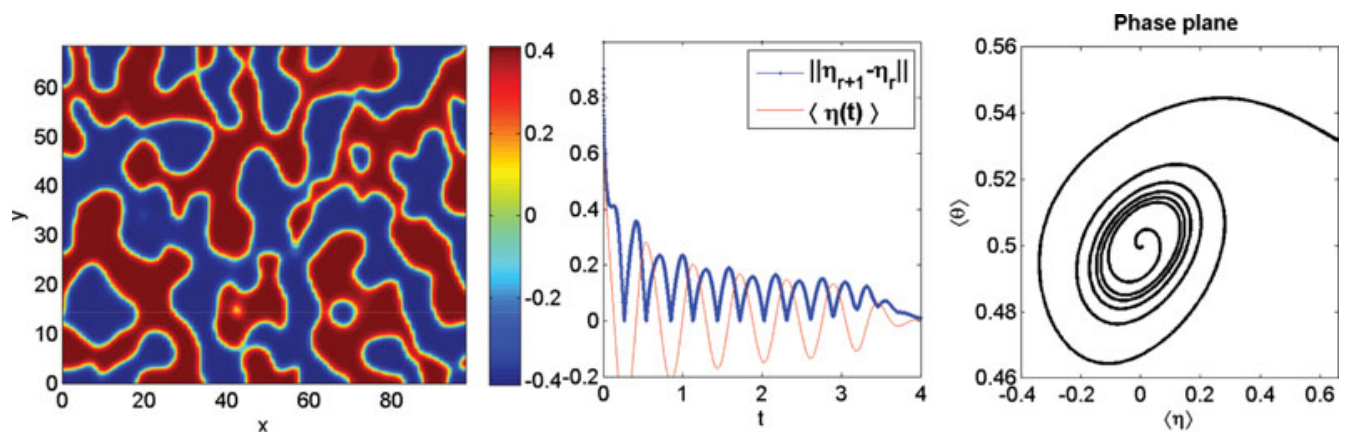

FIGURE 12. Spatio-temporal dynamics in the Hopf region, near the Hopf bifurcation line in the case $d=22,735$. The numerical values for the bifurcation parameters are: $B=109, C=2.794$. Left panel: asymptotic stationary pseudo-labyrinthine pattern. Middle panel: spatially averaged value $\langle\eta(t)\rangle$ until $T_{f}=4$. Right panel: corresponding dynamics in the phase plane $(\langle\eta\rangle,\langle\theta\rangle)$, where a stable focus is attained.

$d$, the corresponding Turing Region $R_{1}$ becomes larger and then the B-coordinate of the $\mathrm{TH}$ point increases (see Figure $2(\mathrm{~b})$ ). The above results thus suggest that by moving in the Hopf region - close to the Hopf line - homogeneous oscillatory behaviour prevails near the TH point, whereas stationary spatial organization can be found moving away from the TH point along the Hopf line.

\subsection{Oscillating Turing patterns}

In this subsection, we propose a numerical validation of the analytical findings of Section 6 regarding TH instabilities of the spatially uniform periodic solution. To better elucidate the relevant phenomenology, we scale the adimensional model (2.1) by introducing new length and time scales: $\mathbf{r}=\sqrt{\rho} \mathbf{r}^{\prime}, t=\rho t^{\prime}$.

Dropping the primes, the scaled reaction-diffusion system becomes

$$
\begin{aligned}
& \frac{\partial \eta}{\partial t}=\Delta \eta+\rho f(\eta, \theta), \\
& \frac{\partial \theta}{\partial t}=d \Delta \theta+\rho g(\eta, \theta),
\end{aligned}
$$

with $f$ and $g$ given by (2.2) and $\rho$ a positive constant. The scale parameter $\rho$ can be interpreted as proportional to the area of the spatial 2D domain: in this sense, doubling the domain size is equivalent to multiplying the original $\rho$ by 4 . In a more intuitive interpretation [51], $\rho$ may also be regarded as the relative strength of the reaction terms. We fix $d=20$ and the other parameter values as indicated in the caption of Figure 2. We stress that the introduction of the scale parameter has no impact on the TH point that, for the chosen set of parameter values, has coordinates $\left(C_{T H}, B_{T H}\right)=(2.8061,109.13)$. Since we want to investigate the system behaviour near the point $T H$, in the following we set $C=2.794$ and $B=109$. 
First of all, we observe that for system (7.1) with reaction terms given by (2.2), the quantities $\tau_{T}$ and $\delta_{T}$ can be explicitly written as

$$
\tau_{T}=\rho \tau_{e}-\lambda_{k}(1+d), \quad \delta_{T}=\rho^{2} \delta_{e}+\lambda_{k}^{2} d-\lambda_{k} \rho\left[J_{22}^{e}+d J_{11}^{e}\right]
$$

where $\tau_{e}=A_{1}(1-\alpha)-C F_{2}(\alpha, \gamma), \delta_{e}=C\left[B F_{1}(\alpha, \gamma)\left(k_{2}-k_{3}\right)-A_{1} F_{2}(\alpha, \gamma)(1-\alpha)\right]$ and $J_{i j}^{e}$ and $F_{i}(\alpha, \gamma)$ given by (3.1) and (3.2), respectively.

We take $\Omega=[0, l] \times[0, m]$ as spatial domain, where the non-dimensional lengths satisfy $l>m$, such that the smallest positive spatial eigenvalue is given by $\lambda_{(1,0)}=\pi^{2} / l^{2}$.

Hence

$$
\tau_{T} \leqslant 0 \quad \Longleftrightarrow \quad \rho \leqslant \frac{\pi^{2}(d+1)}{l^{2} \tau_{e}} .
$$

For strong instability to occur, condition $\tau_{T}>0$ must hold. To test this case, we choose $l=L_{x}=100$ and $m=L_{y}=70<l$. For strong instability, it is required that $\rho>0.9595$ : we set $\rho=50$. Therefore $\tau_{T}=1.0593$ and $\tau_{T}^{2}-4 \delta_{T}=-4,48,856$, so that a strong $\mathrm{TH}$ instability is expected, associated with $\lambda_{(1,0)}$. This implies the appearance of an intermittent pattern whose theoretical frequency is $\sigma_{i}^{(20)}=529.5085$ that is different from the frequency $\omega_{\text {cycle }}=529.9987$ of the limit cycle obtained via supercritical Hopf bifurcation. Figure 13 shows the numerical simulation for the strong TH instability for $d=20$, $\rho=50$ on the spatial domain $\Omega=[0,100] \times[0,70]: 3 \mathrm{D}$ snapshots of the solution every $\Delta t=0.5$ until $T_{f}=4.5$ show that the spatial structure oscillates both in space and time. Figure 13(b), shows the oscillating behaviour of the space integral $\langle\eta(t)\rangle$ (left panel) and the corresponding limit cycle in the phase plane $(\langle\eta(t)\rangle,\langle\theta(t)\rangle)$ (right panel).

As far as the weak TH instability is concerned, Theorem 6.1 states that this type of instability can occur if one of the following conditions hold: (a) $\tau_{T}>0$ and $\tau_{T}^{2}-4 \delta_{T} \geqslant 0$, (b) $\tau_{T} \leqslant 0$ and $\delta_{T}<0$. To illustrate such phenomenology, we set $l=2$. For the chosen parameter values it is easy to verify that condition (b) is the only choice for weak TH instability and that it is satisfied for $0.9623<\rho<1.1281$. The positive values of $\lambda_{k}$ for which $\delta_{T}<0$ belong to the open interval $\Lambda=\left(\lambda_{-}, \lambda_{+}\right)$, with $\delta_{T}\left(\lambda_{ \pm}\right)=0, \lambda_{ \pm}$given by [55]

$$
\lambda_{ \pm}=\rho \frac{\left(d J_{11}^{e}+J_{22}^{e}\right) \pm \sqrt{\left(d J_{11}^{e}+J_{22}^{e}\right)^{2}-4 d \delta_{e}}}{2 d}
$$

and $d J_{11}^{e}+J_{22}^{e}>0$. By choosing $\rho=1$, we get $\tau_{T}=-51.794<0$ and $\delta_{T}=-0.54<0$. Moreover, $\Lambda=(2.1871,2.5639)$ and $\lambda_{(1,0)}=\frac{\pi^{2}}{l^{2}}=2.4674 \in \Lambda$. In this case, weak TH instability is expected which could lead to a dominant steady pattern slightly oscillating only in time with the same frequency of the limit cycle. However, although condition (b) is met for the occurrence of weak TH instability, numerical investigations carried out for the related values of the parameters have not captured this phenomenology. We find in fact, as the asymptotic pattern, the spatially homogeneous limit cycle forming because of the supercritical Hopf bifurcation. This is likely due to the smallness of the $\Lambda$ set and to the fact that, for this case, there is a very narrow range of the parameter values that satisfy the weak TH instability. 

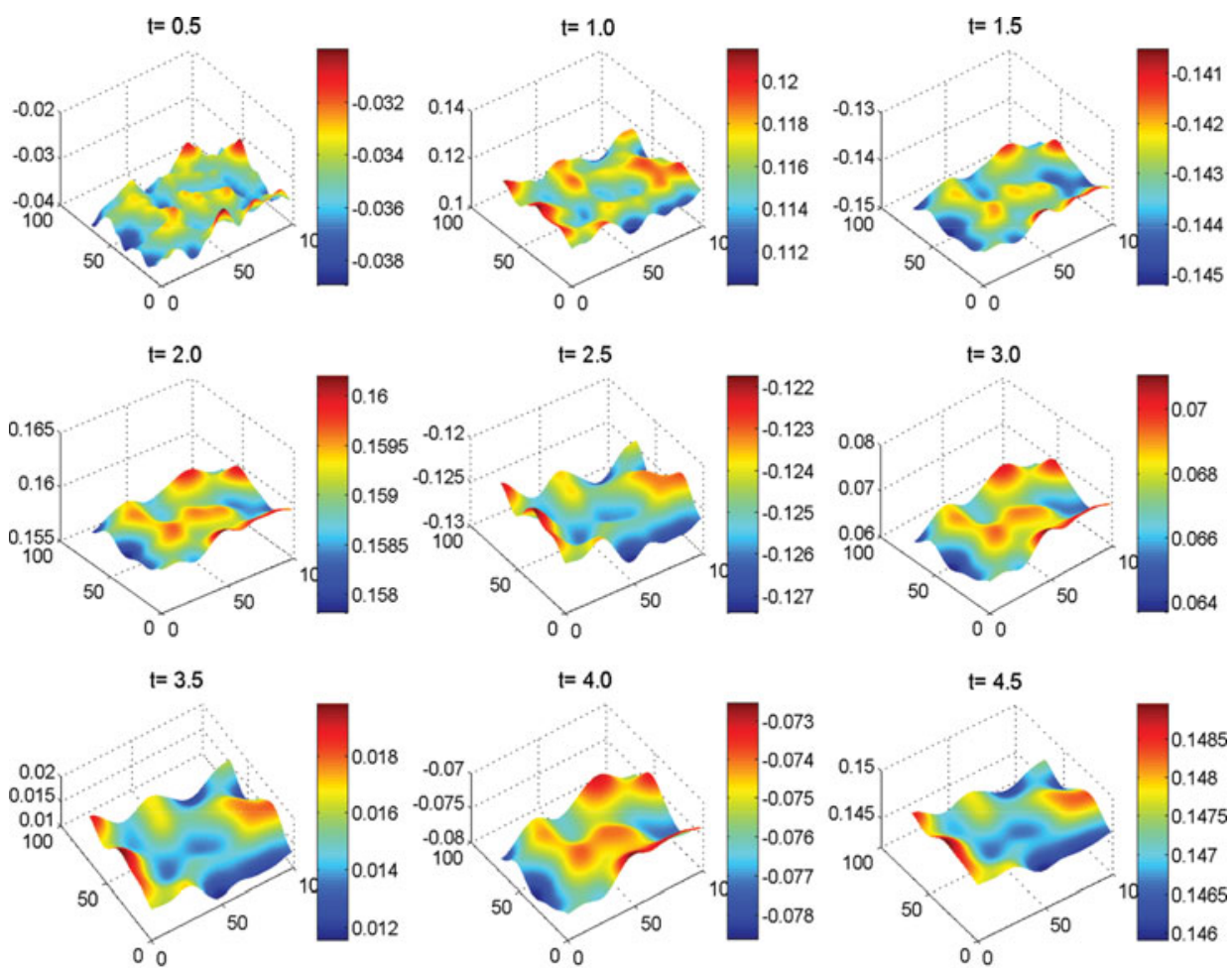

(a)
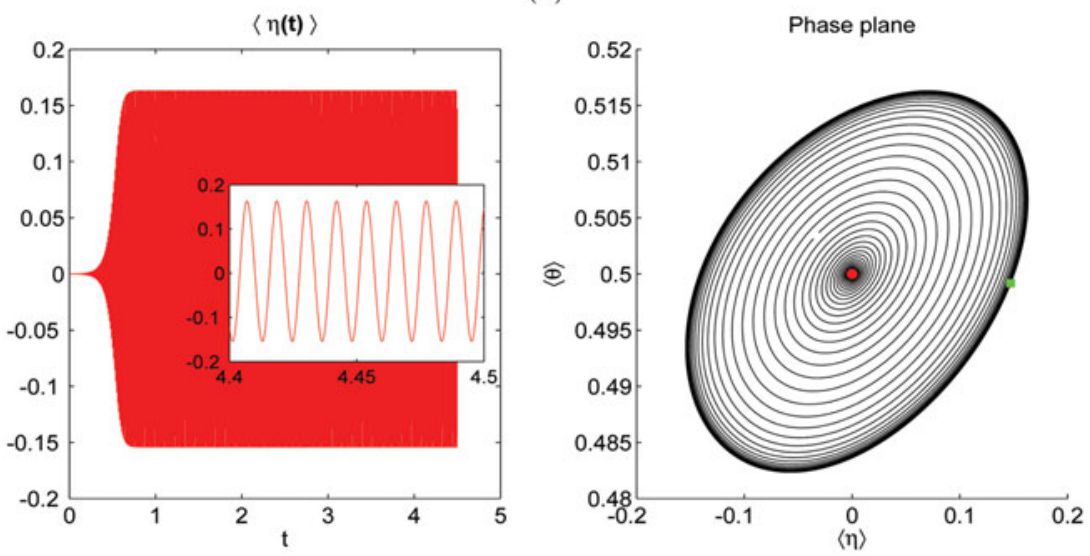

(b)

FIGURE 13. Strong TH instability, obtained for $d=20, \rho=50, B=109, C=2.794, \Omega=$ $[0,100] \times[0,70]$. (a) Snapshots of $3 \mathrm{D}$ spatial patterns every $\Delta t=0.5$ until $T_{f}=4.5$. Oscillations both in space and time are evident looking at the colourbars and comparing the spatial structures of the snapshots. (b) Left panel: spatially averaged value $\langle\eta\rangle$ as a function of time until $T_{f}=4.5$; the inset highlights the periodical behaviour with maximum and minimum values ca. \pm 0.16 . (b) Right panel: the corresponding dynamics in the $(\langle\eta\rangle,\langle\theta\rangle)$ phase plane. The resulting limit cycle is attained starting from the initial conditions $\left(\eta_{0}, \theta_{0}\right)$ (red internal spot) that are spatially random perturbations of the equilibrium $P_{e}$. The (green) * on the limit cycle corresponds to the final time $T_{f}=4.5$. 


\section{Concluding remarks}

In this paper, we investigated the emergence of spatio-temporal organization phenomena for the morphochemical electrodeposition model introduced and experimentally validated in [15]. Our theoretical and numerical findings confirm the extreme flexibility of this mathematical model that is able to account for a great variety of spatial patterns found experimentally. For suitable choices of the parameter values, we highlighted the following scenarios: (i) oscillatory behaviour due to a supercritical Hopf bifurcation; (ii) spatial pattern formation due to Turing instability; (iii) oscillatory spatial patterns due to an interesting interplay between Turing and Hopf instabilities.

We analysed the system behaviour in the neighbourhood of the codimension-two TH bifurcation point by exploiting the analytical approach developed in [55] where the study of the diffusive instability of the spatially uniform periodic solution was applied to the classical Schnakenberg reaction-diffusion model. In this sense, we have also offered a validation of the Ricard-Mischler procedure with a model exhibiting a more complex and general kinetics with respect to the Schnakenberg one.

Numerical challenges related to the simulations of the above scenarios - high accuracy in the space approximation, longtime integration until asymptotic structures, tracking of highly oscillating solutions - have been faced by using the ADI-ECDF schemes introduced in [63] for stationary pattern. Thanks to this approach, that in this paper approximates implicitly the diffusion term and explicitly the reaction terms, the showed simulations have been obtained by using moderate stepsizes both in space and time with consequently reduced computational costs.

Our results also provide a contribution to the debate on the possible emergence of complex behaviours in the neighbourhood of the codimension-two $\mathrm{TH}$ point. In fact, for our model, near the TH point no instances of spatio-temporal chaos have been observed. In particular, when the system parameters approach the $\mathrm{TH}$ point from inside the Turing region, we found that the stationary Turing patterns organize as labyrinthine or tessellation patterns. Outside the Turing Region and in close vicinity of the TH point, we found - in agreement with the phenomenology described in [55] - an intermittent switching between complementary spatial patterns, denominated "strong TH instability". We have, however, found complex spatio-temporal behaviour for this system, but not in the immediate vicinity of the TH point and for parameter choices well inside the Hopf region, where the local system kinetics exhibits an oscillatory regime. This phenomenology appears to be related to the system capability to support spiral wave behaviour in the Hopf region as well as an intriguing phenomenon of spiral break up, that we have pointed up in a recent paper [41].

Although a great number of studies have been performed to investigate the spatiotemporal dynamics of chemical systems in the neighbourhood of a $\mathrm{TH}$ bifurcation point, to the best of our knowledge, our paper provides the first example of such theoretical investigations in the context of alloy electrodeposition, with a particular focus on the arising of oscillating Turing patterns. As shown in [15], our system can be considered as one of the few reaction-diffusion models in morphochemistry capable of developing patterns in very good accordance with the experiments. As a consequence, the theoretical findings obtained in this study are likely to have a very strong impact in the development of 
electrochemical pattern formation in alloy plating in view of control issues and functional applications. Moreover, in order to get an as complete as possible picture of the system spatio-temporal behaviour close to the codimension-two $\mathrm{TH}$ point, we are planning to study system dynamics near the $\mathrm{TH}$ point on the basis of amplitude equations that account for the coupling between a steady Turing-type mode and a Hopf mode. In fact, possible resonances between the Turing and Hopf modes and their harmonics can give rise to new spatio-temporal patterns. In this regard, we aim to derive conditions, expressed in terms of the systems parameters, so that our model can exhibit a stable 2D TH mixed mode as well as other kinds of mixed modes that can emerge as a result of sub-harmonic instabilities.

We finally stress that the rich temporal and spatial dynamics entailed by equation (2.1) and highlighted in this work have a notable bearing on the rationalization of crucial aspects of $3 \mathrm{D}$ electrodeposit morphology. In fact, the discovery of $\mathrm{TH}$ instabilities provides a natural framework - free of ad-hoc physical assumptions and without need of dedicated numerical machinery - for the prediction and treatment of dendritic morphologies with undercuts. Moreover, the morphological periodicities combine in the time evolution of the electrodeposition profile in a way that is able to capture many features of unstable growth shapes found experimentally. In particular, the existence of strong TH instabilities opens up the possibility of achieving a time-averaged smoothing behaviour in electrodeposition regimes exhibiting instantaneous roughening, with notable potential impact on practical metal plating processes, in line with our findings of [12], but based on a different physical mechanism, that can be controlled by both electrical and chemical means.

\section{Acknowledgements}

The present work has been performed under the auspices of the Italian National Group for Mathematical Physics (GNFM-Indam), granted scientific project entitled "Analisi e controllo di strutture morfologiche spaziali in modelli di tipo reazione-diffusione con applicazioni industriali". We also thank the anonymous referees and the handling Editor for their valuable comments and remarks.

\section{References}

[1] Aragon J. L., Barrio R. A., Woolley T. E., Baker R. E. \& Maini P. K. (2012) Nonlinear effects on Turing patterns: Time oscillations and chaos. Phys. Rev. E 86, 026201, 1-4.

[2] Banerjee M. \& Petrovskil S. (2011) Self-organised spatial patterns and chaos in a ratiodependent predator-prey system. Theor. Ecol. 4, 37-53.

[3] Baurmann M., Gross T. \& Feudel U. (2007) Instabilities in spatially extended predator-prey systems: Spatio-temporal patterns in the neighbourhood of Turing-Hopf bifurcations. $J$. Theor. Biol. 245, 220-229.

[4] Bogoliubov, N. N. \& Mitropolski, Y. A. (1961) Asymptotic Methods in the Theory of Nonlinear Oscillations, Gordon and Breach, New York.

[5] Boissonade J., Dulos E. \& De Kepper P. (1995) Onset and beyond Turing pattern formation. In: R. Kapral \& K. Showalter (editors), Chemical Waves and Patterns, Kluwer Academic Publishers, Dordrecht. 
[6] Borckmans P., Dewel G., De Wit A., Dulos E., Boissonade J., Gauffre F. \& De KepPER P. (2002) Diffusive instabilities and chemical reactions. Int. J. Bif. Chaos 12, 23072332.

[7] Bozzini B., Lacitignola D. \& Sgura I. (2008) A reaction-diffusion model of spatial pattern formation in electrodeposition. J. Phys.: Conf. Ser. 96, 012051.

[8] Bozzini B., D’Urzo L., Lacitignola D., Mele C., Sgura I. \& Tondo E. (2009) An investigation into the dynamics of Au electrodeposition based on the analysis of SERS spectral time series. Trans. Inst. Met. Fin. 87, 193-200.

[9] Bozzini B., Lacitignola D. \& Sgura I. (2010) Morphological spatial patterns in a reaction diffusion model for metal growth. Math. Biosci. Eng. 7, 237-258.

[10] Bozzini B., Sgura I., Lacitignola D., Mele C., Marchitto M. \& Ciliberto A. (2010) Prediction of morphological properties of smart-coatings for $\mathrm{Cr}$ replacement, based on mathematical modelling. Adv. Mat. Res. 138, 93-106.

[11] Bozzini B., Lacitignola D. \& SGURA I. (2011) Travelling waves in a reaction-diffusion model for electrodeposition. Math. Comput. Simul. 81, 1027-1044.

[12] Bozzini B., Lacitignola D. \& SGuRa I. (2011) Frequency as the greenest additive for metal plating: Mathematical and experimental study of forcing voltage effects on electrochemical growth dynamics. Int. J. Electrochem. Sci. 6, 4553-4571.

[13] Bozzini B., Lacitignola D., Mele C. \& Sgura I. (2012) Coupling of morphology and chemistry leads to morphogenesis in electrochemical metal growth: A review of the reactiondiffusion approach. Acta Appl. Math. 122, 53-68.

[14] Bozzini B., Lacitignola D., Mele C. \& Sgura I. (2012) Morphogenesis in metal electrodeposition. Note di Matem. 32, 7-46.

[15] Bozzini B., Lacitignola D. \& SGURa I. (2013) Spatio-temporal organisation in Alloy electrodeposition: A morphochemical mathematical model and its experimental validation. $J$. Solid State Electrochem. 17, 467-479.

[16] Mele C., Catalano M., Taurino A. \& Bozzini B. (2013) Electrochemical fabrication of NPGsupported manganese oxide nanowires based on electrodeposition from eutectic urea/choline chloride ionic liquid. Electrochim. Acta 87, 918-924.

[17] Bozzini B., Gianoncelli A., Mele C. \& Kiskinova M. (2013) Electrochemical fabrication of nanoporous gold decorated with manganese oxide nanowires from eutectic urea/choline chloride ionic liquid. Part II - Electrodeposition of Au-Mn: A study based on soft X-ray microspectroscopy. Electrochim. Acta, 114, 889-896.

[18] Castets V., Dulos E., Boissonade J. \& De Kepper P. (1990) Experimental evidence of a sustained standing Turing-type nonequilibrium chemical pattern. Phys. Rev. Lett. 64, 2953.

[19] Crawford J. D. (1991) Introduction to bifurcation theory. Rev. Mod. Phys. 63, 991-1037.

[20] Cross M. C. \& Honenberg P. C. (1993) Pattern formation outside the equilibrium. Rev. Mod. Phys. 65, 851-1112.

[21] Cross M. \& Greenside H. (2009) Pattern Formation and Dynamics in Nonequilibrium Systems, Cambridge University Press, Cambridge.

[22] Dewel G., Borckmans P., De Wit A., Rudovics B., Perraud J., Dulos E., Boissonade J. \& KePPER P. D. (1995) Pattern selection and localized structures in reaction-diffusion systems, Physica A 213, 181-198.

[23] Dewel G., De Wit A., Metens S., Verdasca J. \& Borckmans P. (1996) Pattern selection in reaction-diffusion systems with competing bifurcations. Phys. Scr. T67, 51-57.

[24] De Wit A., Lima D., Dewel G. \& Borckmans P. (1996) Spatiotemporal dynamics near a codimension-two point. Phys. Rev. E 54, 261-271.

[25] De Wit A. (1999) Spatial patterns and spatiotemporal dynamics in chemical systems. Adv. Chem. Phys. 109, 435-513.

[26] Dobrovolska Ts., Krastev I., Zabinski P., Kowalik R. \& Zielonka A. (2011) Oscillations and self-organization phenomena during electrodeposition of silver-indium alloys. Experimental study. Arch. Metall. Mater. 56, 645-657. 
[27] Dobrovolska Ts., Lopez-Sauri D. A., Veleva L. \& Krastev I. (2012) Oscillations and spatio-temporal structures during electrodeposition of $\mathrm{AgCd}$ alloys. Electrochim. Acta 79, $162-169$.

[28] Epstein J. M. (1997) Nonlinear Dynamics, Mathematical Biology and Social Science, AddisonWesley, Reading, MA.

[29] Gambino G., Lombardo M. C. \& Sammartino M. (2012) Turing instability and traveling fronts for a nonlinear reaction-diffusion system with cross-diffusion. Math. Comput. Simul. 82, 1112-1132.

[30] Gambino G., Lombardo M. C. \& Sammartino M. (2013) Pattern formation driven by crossdiffusion in a 2D domain. Nonlinear Anal. Real World Appl. 14, 1755-1779.

[31] Gross T. \& Feudel U. (2006) Generalized models as a universal approach to the analysis of nonlinear dynamical systems. Phys. Rev. E 75, 1-14.

[32] Hoyle R. (2006) Pattern Formation. An Introduction to Methods, Cambridge University Press, Cambridge.

[33] Just W., Bose M., Bose S., Engel H. \& Scholl E. (2001) Spatiotemporal dynamics near a supercritical Turing-Hopf bifurcation in a two-dimensional reaction-diffusion system. Phys. Rev. E 64, 1-12.

[34] Kaminaga A., Vanag V. K. \& Epstein I. R. (2005) 'Black spots' in a surfactant-rich BZ-AOT microemulsion system. J. Chem. Phys. 122, 174706, 1-11.

[35] Kapral R. \& Showalter, K. (1995) Chemical Waves and Patterns, Kluwer Academic Publishers, Dordrecht.

[36] Koper M. T. M. (1998) Non-linear phenomena in electrochemical systems. J. Chem. Soc. Faraday Trans. 94, 1369-1378.

[37] Krastev I. \& Koper M. T. M. (1995) Pattern formation during the electrodeposition of a silver-antimony alloy. Physica A 213, 199-208.

[38] Krastev I. (2009) Self-structured silver alloy coatings and their properties. J. Eng. Process. Manage. 1, 104-112.

[39] Krastev I., Dobrovolska Ts., Lacnjevac U. \& Nineva S. (2012) Pattern formation during electrodeposition of indium-cobalt alloys. J. Solid State Electrochem. 16, 34493456.

[40] Krischer K., Mazouz N. \& Grauel P. (2001) Fronts, waves, and stationary patterns in electrochemical systems. Angew. Chem. Int. Ed. 40, 850-869.

[41] Lacitignola D., Bozzini B. \& Sgura I. (2014) Spatio-temporal organization in a morphochemical electrodeposition model: Analysis and numerical simulation of spiral waves. Acta Appl. Math., 132, 377-389.

[42] Lee K. J., McCormick W. D., Ouyang Q. \& Swinney H. L. (1993) Pattern Formation by Interacting Chemical Fronts. Sci. New Ser. 261(5118), 192-194.

[43] Leppanen T., Karttunen M., Kaski K. \& Barrio R. A. (2004) Turing systems as models of complex pattern formation. Braz. J. Phys. 34, 368-372.

[44] Leppänen T., Karttunen M., Barrio R. A. \& Kaski K. (2004) Morphological transitions and bistability in Turing systems. Phys. Rev. E 70, 066202.

[45] Li Y-J., Oslonovitch J., Mazouz N., Plenge F., Krischer K. \& Ertl G. (2001) Turing-type patterns on electrode surfaces. Science 291, 2395-2398.

[46] Maini P. K. \& Othmer H. G. (2001) Mathematical models for biological pattern formation. In: The IMA Volumes in Mathematics and its Applications - Frontiers in Applications of Mathematics, Vol. 121, Springer, New York.

[47] MAINI P. K. (2004) Using mathematical models to help understand biological pattern formation. C. R. Biol. 327, 225-234.

[48] Malchow H., Petrovskii S. V. \& Venturino E. (2008) Spatiotemporal Patterns in Ecology and Epidemiology, Chapman \& Hall, U.K.

[49] Meixner M., De Wit A., Bose S. \& Scholl E. (1997) Generic spatiotemporal dynamics near codimension-two Turing Hopf bifurcations. Phys. Rev. E 55, 66906697. 
[50] Miguez D. G., Alonso S., Munuzuri A. P. \& Sagues F. (2006) Experimental evidence of localized oscillations in the photosensitive chlorine dioxide-iodine-malonic acid reaction. Phys. Rev. Lett. 97, 178301, 1-4.

[51] Murray J. D. (2002) Mathematical Biology II; Spatial Models and Biomedical Applications, 3rd ed., Springer, New York.

[52] Orbán M., Kurin-Csörgei K., Zhabotinsky A. M. \& Epstein I. R. (2001) A new chemical system for studying pattern formation: Bromate-Hypophosphite-Acetone - dual catalyst. Faraday Disc. 120, 11-19.

[53] Ouyang Q. \& Swinney H. L. (1991) Transition from a uniform state to hexagonal and striped Turing patterns. Nature 352, 610-612.

[54] Perraud J. J., Agladze K., Dulos E. \& De Kepper P. (1992) Stationary Turing patterns versus time-dependent structures in the chlorite-iodide-malonic acid reaction. Physica $A \mathbf{1 8 8}$, $1-16$.

[55] Ricard M. R. \& Michler S. (2009) Turing Instabilities at Hopf bifurcation. J. Nonlinear Sci. 19, 467-496.

[56] Rovinsky A. \& Menzinger M. (1992) Interaction of Turing and Hopf bifurcations in chemical systems. Phys. Rev. A 46, 6315-6322.

[57] Rudovics B., Dulos E. \& De KePper P. (1996) Standard and nonstandard Turing patterns and waves in the CIMA reaction. Phys. Scr. T67, 43-50.

[58] F. Sagú́s \& Epstein I. R. (2003) Nonlinear chemical dynamics. Dalton Trans. 1201-1217.

[59] Saitou M. \& FukuOKa Y. (2004) An experimental study on stripe pattern formation of Ag-Sb electrodeposits. J. Phys. Chem. B 108, 5380-5385.

[60] Saitou M. \& Fukuoka Y. (2004) A study on transient changes of surface morphologies in Ag and $\mathrm{Sb}$ coelectrodeposition. J. Electrochem. Soc. 151, C627-C632.

[61] Saitou M. \& Fukuoka Y. (2005) Stripe patterns in Ag-Sb co-electrodeposition. Electrochim. Acta 50, 5044-5049.

[62] Sanders, J. A. \& Verhulst, F. (1985) Averaging Methods in Nonlinear Dynamical Systems, Applied Mathematical Sciences, Vol. 59, Springer, New York.

[63] Sgura I., Bozzini B. \& Lacitignola D. (2012) Numerical approximation of Turing patterns in electrodeposition by ADI methods. J. Comput. Appl. Math. 236, 4132-4147.

[64] Sgura I., Bozzini B. \& Lacitignola D. (2012) Numerical approximation of oscillating Turing patterns in a reaction-diffusion model for electrochemical material growth. AIP Conf. Proc. 1493, 896-903.

[65] Sherratt, J. A. (2012) Turing patterns in deserts. In: S. B. Cooper, A. Dawar \& B. Lowe (editors), How the World Computes, Lecture Notes in Computer Science, Vol. 7318, Springer, New York, pp. 667-674.

[66] Turing A. M. (1952) The chemical bases of morphogenesis, Phil. Trans. R. Soc. London B 237, $37-72$.

[67] Vanag V. K. \& Epstein I. R. (2008) Design and control of patterns in reaction-diffusion systems. Chaos 18, 026107, 1-11.

[68] VanaG V. K. \& Epstein I. R. (2009) Pattern formation mechanisms in reaction-diffusion systems. Int. J. Dev. Biol. 53, 673-681.

[69] Yang L., Dolnik M., Zhabotinsky A. M. \& Epstein I. R. (2002) Spatial resonances and superposition patterns in a reaction-diffusion model with interacting Turing modes. Phys. Rev. Lett. 88, 208303, 1-4.

[70] Yang L. \& EPSTEIN I. R. (2003) Oscillatory Turing patterns in reaction-diffusion systems with two coupled layers. Phys. Rev. Lett. 90, 178303, 1-4. 